\title{
On the dynamic information underlying visual anticipation skill
}

\author{
RAOUl HuYs \\ University of the Mediterranean, Marseille, France \\ NichOLAS J. SMEETON \\ Liverpool John Moores University, Liverpool, England \\ and University of Brighton, Eastbourne, England \\ Nicola J. Hodges \\ University of British Columbia, Vancouver, British Columbia, Canada \\ Peter J. Beek \\ Vrije Universiteit Amsterdam, Amsterdam, The Netherlands \\ AND \\ A. Mark Williams \\ Liverpool John Moores University, Liverpool, England
}

\begin{abstract}
What information underwrites visual anticipation skill in dynamic sport situations? We examined this question on the premise that the optical information used for anticipation resides in the dynamic motion structures, or modes, that are inherent in the observed kinematic patterns. In Experiment 1, we analyzed whole-body movements involved in tennis shots to different directions and distances by means of principal component analysis. The shots differed in the few modes that captured most of the variance, especially as a function of shot direction. In Experiments 2 and 3, skilled and less skilled tennis players were asked to anticipate the direction of simulated shots on the basis of kinematic patterns in which only the constituent dynamic structures were manipulated. The results indicated that players predicted shot direction by picking up the information contained in multiple lowdimensional dynamic modes, suggesting that anticipation skill in tennis entails the extraction of this dynamic information from high-dimensional displays.
\end{abstract}

Biological motion patterns contain information about the agent's identity, emotion, and intentions. For instance, people can discern the gender of someone walking in the absence of obvious cues (Troje, 2002). Emotions like anger, disgust, fear, happiness, and sadness are readily gleaned from bodily movements (Dittrich, Troscianko, Lea, \& Morgan, 1996). Similarly, skilled tennis and squash players can accurately predict where an opponent will play the ball before it is actually hit (Abernethy, 1990; Williams, Ward, Knowles, \& Smeeton, 2002). In these latter examples, well-trained individuals (i.e., domain-specific experts) are more proficient in "reading" the relevant information from an unfolding action than novice observers are. This skillspecific feature is particularly well documented for racket sports (see, e.g., Smeeton, Williams, Hodges, \& Ward, 2005; Williams, Davids, \& Williams, 1999).

In racket sports such as tennis, squash, and badminton, expert players are typically faster and/or more accurate in predicting the outcome of an opponent's action (e.g., the direction and/or depth of a serve) than less skilled players are (Abernethy, 1990; Abernethy \& Russell, 1987; Williams et al., 2002). In studies of this phenomenon, participants are usually presented with video clips showing the execution of strokes performed by an opponent. The clip is then stopped at particular time points during the unfolding action, and participants are invited to indicate the outcome of the action, such as the stroke direction and/or depth, or the ball or shuttlecock's future landing position. To investigate the importance of certain time windows and/or body parts for anticipation, researchers have used temporal and/ or spatial occlusion paradigms, as well as eye movement registration techniques (for reviews, see Williams et al., 1999; Williams \& Ericsson, 2005).

Abernethy and Russell (1987) examined the anticipation accuracy of novice and expert players who were presented with a series of video clips of badminton strokes that were edited so that increasing portions of the event were visible or different body parts of the opponent were occluded.

R. Huys, raoul.huys@univmed.fr 
In trials in which clips of the full shot were shown, participants' eye movements were recorded. The experts were able to use information arising earlier in the action than novices were, and anticipation performance deteriorated when either the arm or the arm and racket were occluded. Since the visual search strategies of both skill groups did not differ, the authors concluded that perceptual skill is based on the ability to make full use of the available information. These results have been corroborated by other researchers, confirming that experts are able to pick up and use information arising earlier in an action sequence than novices are (see, e.g., Abernethy, 1990; Abernethy, Gill, Parks, \& Packer, 2001; Ward, Williams, \& Bennett, 2002). Moreover, unlike novices, who rely primarily on arm and racket movement, experts pick up more information from proximal body regions, such as the hips and shoulders (Ward et al., 2002; Williams et al., 2002).

Although researchers have revealed when and where information can be picked up in order to facilitate anticipation, the methods employed for this purpose were ill suited to uncover what information performers may use. Anticipation is only possible if one or more invariant features exist that differentiate between competing actions, be they squash strokes or passing shots in tennis. Previously, researchers have suggested that dynamic features, rather than anatomical or physical ones, form the informational basis underlying the perception of biological motion (Runeson \& Frykholm, 1983; Troje, 2002; Westhoff $\&$ Troje, 2007). The detailed investigation of similarities and/or dissimilarities in the dynamics of different actions, such as squash or tennis shots that vary in depth and direction, is therefore a prerequisite for coming to terms with the perceptual basis of visual anticipation skills, an endeavor that, to the best of our knowledge, has not yet been pursued (but see Troje, 2002, and Haken, 2000, 2004, for similar approaches to event recognition and pattern recognition, respectively). In the present research, we identify the dynamic structures underlying the execution of distinct tennis shots using the Karhunen-Loève expansion, also referred to as principal component analysis (PCA). In this context, it should be noted that, whereas dynamics is used in classical mechanics in reference to the causal relation between forces and the resulting motion (i.e., as a synonym for kinetics), we use it to refer more broadly to all time-evolving phenomena, including time-dependent motion structures (Newell, Liu, \& Mayer-Kress, 2001; Strogatz, 1994). Having identified the dynamic structures of interest, we examine the significance of these structures for visual anticipation skill in two experiments. Before introducing our specific expectations with regard to the outcome of these experiments, we briefly discuss previous insights into the information used for biological motion perception and anticipation, as well as recent methodological advances in uncovering this information.

\section{THE PERCEPTION OF BIOLOGICAL MOTION}

Swedish researchers (Johansson, 1973, 1976; Runeson, 1977/1983; Runeson \& Frykholm, 1981, 1983) undertook the initial attempts at pinpointing the information used in biological motion perception. In a series of experiments, naive observers were shown humans performing actions in point-light display (PLD) format. Points of lights were presented corresponding to the location of anatomical landmarks on the body (e.g., shoulders, elbows, and hips) against a homogeneous dark background. When viewing moving point-light images, observers were able to recognize actions such as walking (Johansson, 1973, 1976) or lifting a weight (Runeson \& Frykholm, 1981, 1983), as well as an actor's gender and the intention to obscure the weight of a lifted object (Runeson \& Frykholm, 1983).

PLDs have subsequently been used to examine anticipation in sports. Ward et al. (2002) studied the ability of experienced and less experienced tennis players to anticipate an opponent's intentions from normal film, as well as from PLDs. The experienced players anticipated more quickly and more accurately than the less experienced players. Although performance deteriorated when participants viewed PLDs relative to film, both groups still performed above chance levels, and the experienced participants maintained their superiority over their less experienced counterparts (see also Abernethy et al., 2001). In a similar vein, Shim, Carlton, Chow, and Chae (2005) examined anticipation accuracy as a function of skill and display type through the use of a live model, filmed images, and PLDs. They reported that response accuracy was above chance levels in all display conditions. However, a significant interaction between skill and display type indicated that the experts' response accuracy decreased when they viewed a PLD as opposed to film and live-model presentation modes; surprisingly, the reverse was observed in the novice group, who improved moderately when the display type moved from live model to film and then PLDs, respectively. In fact, the skill difference was no longer significant under the latter condition. Shim et al. suggested that the skilled players, in contrast with the novices, were able to extract contextual information, or subtle visual clues, from the live-model and film-based displays, as well as from the relative motion presented in the PLDs. They also suggested that this surplus information was used in an additive fashion (cf. Bruno \& Cutting, 1988).

The findings from these studies suggest that the temporal structure inherent in the execution of tennis shots informs observers about the ball's future landing position before it is actually hit, but they do not address the question of how information is embodied in the motion patterns. This issue was tackled in the context of gender recognition by Troje (2002), who used PCA to decompose male and female walking patterns into four rhythmical components that together captured more than $98 \%$ of the variance of the original 45-dimensional time series. These four components were used to classify and synthesize male and female walking patterns. Troje invited participants to judge the gender of walkers whose walking patterns were simulated using dynamic information, motion-mediated (structural) information (e.g., hip:shoulder ratios), or both, and found that the dynamics of the motion proved more informative for gender classification than did the motionmediated structural cues. The combination of dynamic 
and structural information did not improve performance relative to dynamic information only.

In the present study, we used PCA to examine wholebody movements in three (Cartesian) directions of different passing shots in tennis. Our aim was twofold. First, we wanted to identify potential differences in the dynamic structures (coordination patterns) underlying the execution of different passing shots. Second, we wanted to verify the importance of these structures for anticipation by manipulating their availability to observers. In the first part of this article, we present the results of the PCA on different passing shots in tennis, followed by a discussion culminating in specific predictions about the dynamics that could be critical for the anticipation of these shots. In the second part of the article, we present two experiments that were designed and conducted to test the predictions derived from the PCA.

\section{EXPERIMENT 1}

The execution of a specific action varies between individuals, as well as within an individual, across attempts (Bernshtel̆n, 1967; Kelso, 1995; Scholz \& Schöner, 1999). It seems reasonable to expect that in the execution of tennis shots, body areas that supposedly contribute relatively little to passing accuracy will exhibit a larger variability across trials than will the end-effector and mechanically linked areas (i.e., the shoulder-arm-racket linkage). Similarly, body areas that move consistently from one shot to the next (for similar directions and distances) are more likely to be relevant to anticipation than those that do not. Findings from eye movement research support this line of reasoning: When attempting to anticipate tennis shots, novices typically focus on the arm and racket; experts additionally focus on the more proximal shoulder and hip areas (Ward et al., 2002; Williams et al., 2002).

In this first, preparatory study, we used PCA to identify systematic similarities and/or dissimilarities in coordination patterns across different passing shots in tennis (in particular, inside-out and cross-court shots to short and deep targets; see Figure 1). Similarities across different shots are expected to manifest themselves via similar eigenvector coefficients, whereas structural dissimilari- ties between these shots (e.g., consistent, distinct hipshoulder coordination for shots to different directions) can be expected to be revealed in consistent differential eigenvector coefficients. Uncorrelated dissimilarities across shots (e.g., varying knee-ankle coordination across shots to the same direction) are expected to present themselves as variable eigenvector coefficients, above all in the (higher) modes that account for only small portions of the variance. We used PCA to separate the structural features of passing shots from the inherent random features (cf. Daffertshofer, Lamoth, Meijer, \& Beek, 2004), resulting in a characteristic description of the passing shots.

We expected the similarities in each combination of inside-out and cross-court shots to short and deep targets to be greater than the dissimilarities; the amount of variance associated with executing any passing shot is in all likelihood considerably larger than that associated with shot-specific adjustments. For example, in all these shots, the torso rotates to support the arm and racket swing up to ball contact. Playing the ball to a particular place probably requires relatively small adjustment of this motion. Therefore, we did not expect to find that there were shotspecific modes, but rather that a few modes would effectively describe all shots. We expected that consistent differential contributions from the time series to the modes (represented in the eigenvector coefficients) would differentiate shot types. Moreover, we expected to find consistent differences between shot types, predominantly in the racket, right arm, shoulder, and hip linkage. Finally, we expected to find fewer dynamic differences in shot distance than in shot direction, because variations in shot distance may well be achieved by means of a scaling of the dynamics.

\section{Method}

\section{Participants}

Six tennis players ( 2 male, 4 female) between 15 and 18 years of age $(M=18.0$ years, $S D=1.1)$ participated. All were right-handed, played competitively at a national level (mean national rating $=$ $4.75, S D=1.40 ; 1.1$ and 10.2 are the highest and lowest ratings, respectively, within the Lawn Tennis Association's [LTA] rating system in the U.K.), and had played an average of $158.3(S D=43.2)$ tournament matches within the preceding year. Participants gave their informed consent prior to taking part in all three experiments,

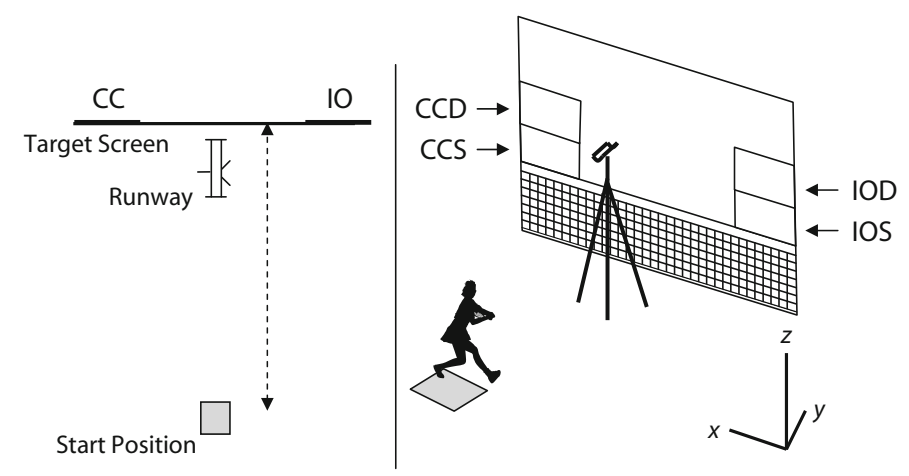

Figure 1. Plan view (left panel) and 3-D view (right panel) of the experimental setup. CC, cross-court; IO, inside-out; $S$, short; D, deep. 
and each experiment was conducted in accordance with the ethical guidelines of the lead institution.

\section{Apparatus}

Four different targets were constructed in the laboratory (see Figure 1) in order to simulate passing shots directed to the right and left side of a tennis court, respectively called inside-out and cross-court shots in tennis terminology. The lower and upper parts of these targets were to simulate short and deep shots (i.e., near and far areas of a tennis court, respectively). The targets, which were $0.40 \times 1.00 \mathrm{~m}$ (height $\times$ width) large, were placed so that the midpoints of the lower (higher) pair were $1.20 \mathrm{~m}(1.60 \mathrm{~m})$ above ground level and $2.50 \mathrm{~m}$ apart from each other. This setup resulted in an accurate representation of a tennis court. Whereas, in principle, shots directed to the deep (short) target may in reality result in a short (deep) shot, observation of the participants' performance, as well as their self-reported performance evaluations, suggested that this was not the case. The participants' start position was a distance of $6 \mathrm{~m}$ from the targets, at the midpoint of and perpendicular to the midline between the targets. In order to standardize ball trajectories prior to the shot, we projected the ball toward the participant using a runway located between the targets at a vertical distance of $2.50 \mathrm{~m}$ above the floor.

Whole-body, 3-D displacement data were recorded at $240 \mathrm{~Hz}$ (spatial accuracy $\leq 1 \mathrm{~mm}$ ) by means of an infrared motion capture system (Qualisys, Sweden), which consisted of six cameras positioned at $45^{\circ}, 90^{\circ}, 135^{\circ}, 225^{\circ}, 270^{\circ}$, and $335^{\circ}$, respectively, to the transverse plane of the player's start position. Spherical retroflective markers (width $=15 \mathrm{~mm}$ ) were placed on the left and right shoulders, elbows, wrists, hips, knees, ankles, and toes, on the top, bottom, left, and right side of the racket face, on the ball, and on two reference points situated on the floor in line with the start position, $3.5 \mathrm{~m}$ apart. The displacement data of the markers that were attached to the body (14) and racket (4) were used for further analysis (see also Figures 4-6). In addition, a Panasonic S-VHS video recorder (Model NV-MS5) was placed about $3 \mathrm{~m}$ behind the start position and was aimed forward, in order to record the targets.

\section{Procedure}

Prior to the experimental trials, each participant practiced several shots to familiarize herself or himself with the task environment Each participant performed 10 forehand strokes to each of the four targets. At the start of a trial, the participant placed herself or himself at the start position. The experimenter indicated verbally the target to be aimed at (inside-out short, inside-out deep, cross-court short, or cross-court deep), after which the ball was projected toward the participant, who struck it using a conventional tennis racket after it had bounced once on the floor.

\section{Data Analysis}

For each participant and condition, the four trials were selected in which the target was hit at a distance closest to its extreme cornerthat is, the upper left (right) corner for a deep cross-court (insideout) shot and the lower left (right) corner for a short cross-court (inside-out) shot. The number of trials we could analyze was limited to four due to the available computational capabilities; the inclusion of a fifth trial resulted in a state vector that was too large to allow for computing its covariance matrix. For each trial, the start point and end point were defined as the initiation of right-wrist backward movement (in the $y$-direction) and the moment of racket-ball contact, respectively. Each trial's duration $\left(T_{t}\right)$ was determined, and each time series standard deviation $\left(T S_{S D}\right)$ was computed. The latter provided an indication of the scaling of the trajectory (and its higher derivatives) of the time series in question. Since we were interested in the scaling features of shots in situ, we did not normalize these standard deviations with respect to shot depth.

We examined the tennis shot executions in terms of the PCA, which is an unbiased statistical method for identifying low-dimensional components in high-dimensional motion patterns (Daffertshofer et al., 2004; Haken, 1996). In particular, unlike the standard statistical application of PCA that is based on the covariance matrix of scalar values - which contains no information about events as they unfold in time-we used PCA to compute the covariance matrix across time series, so that a time evolution would be associated with each eigenvector (mode). In brief, the general aim of PCA is to effectively approximate an $N$-dimensional data set with fewer dimensions $M$. To do so for an arbitrary set of time series $\mathbf{q}(t):=\left[q_{1}(t), q_{2}(t), q_{3}(t), \ldots q_{N}(t)\right]^{T} \cdot \mathbf{e}^{k}$ (where $\mathrm{e}^{k}$ represents the $k$ th basis vector for $k=1 \ldots N ; t=t_{0} \ldots T$ ), one chooses a different set of vectors $\left\{\mathbf{v}^{k}\right\}$ to obtain

$$
\mathbf{q}(t) \approx \sum_{k=1}^{M<N} \xi_{k}(t) \mathbf{v}^{k}
$$

The appropriate choice of the vectors $\mathbf{v}^{k}$ is found by minimizing the least square error:

$$
E_{m}:=\frac{1}{T} \int_{0}^{T}\left[\mathbf{q}(t)-\sum_{k=1}^{M<N} \xi_{k}(t) \mathbf{v}^{k}\right]^{2} d t=\min .
$$

The vectors $\mathbf{v}^{k}$ are assumed to be orthogonal - that is, independent. Algebraically, this procedure is realized by diagonalization of the data's covariance matrix. The eigenvalues $\lambda_{k}$ of the covariance matrix (after being rescaled so that their sum equaled 1) reflect the amount of variance covered by the corresponding eigenvector $\mathbf{v}^{k}$. The $N$ coefficients of each corresponding eigenvector $\mathbf{v}^{k}=v_{i}^{k}(i=1 \ldots N)$ reflect the degree to which each of the time series $i$ contributed to $\mathbf{v}^{k}$-in other words, the degree to which they share a specific time evolution. The eigenvectors, or principal components $\mathbf{v}^{k}$, are often referred to as modes (Haken, 2000), which we will use here also. Finally, the time evolutions $\xi_{k}(t)$ (generally referred to as projections) of each mode $\mathbf{v}^{k}$ can be obtained by the scalar product

$$
\xi_{k}(t)=\mathbf{v}^{k} \cdot \mathbf{q}(t) .
$$

In order to examine whether shots in different directions can be distinguished on the basis of shot-specific modes, the time series of each trial for each participant and condition were resampled to the mean length of all time series, mean subtracted, normalized to unit variance, and combined into an $N$-dimensional state vector $\mathbf{q}(t)(N=4$ [trials] $\times$ 54 [time series] $=216 ; t=t_{\text {start }} \ldots t_{\text {ball contact }}$. Each mode's projection $\xi_{k}(t)$ was established (see Equation 3), and for Modes 1, 2, and 3, the covariance (normalized to the interval $[-1,1]$ ) between corresponding projections was computed. To anticipate, this analysis indicated that shots to different directions were not differentiated by shot-specific modes (see "Shot comparisons across participants" below). Therefore, a new $N$-dimensional state vector $\mathbf{q}(t)$ was constructed - this time including all mean-subtracted and normalized time series from all participants $(N=6$ [participants] $\times 4$ [conditions] $\times 4$ [trials] $\times 54$ [time series $\left.]=5,184 ; t=t_{\text {start }} \ldots t_{\text {ball contact }}\right)$-and subjected to PCA, and the projections $\xi_{k}(t)$ were computed next. Recall that each eigenvector $\mathbf{v}_{i}^{k}(i=1 \ldots N)$ contains $N$ eigenvector coefficients $i$, corresponding to different marker locations in the $x$-, $y$-, and $z$-directions from short and deep inside-out and cross-court shots. These coefficients were analyzed for modes $k=1 \ldots 5$ in terms of marker location, Cartesian direction, shot direction, and shot distance.

$T_{t}, T S_{S D}$, and the eigenvector coefficients $v_{i}^{k}$ corresponding to the marker locations in the $x$-, $y$-, and $z$-directions of the first five modes were subjected to a two-way ANOVA with shot direction (2) and shot distance (2) as within-participants factors. We refrained from subjecting the $T S_{S D}$ to a four-way ANOVA with movement direction and marker location, because we were interested in potential effects of shot direction and shot distance. Whenever the sphericity assumption was violated, the degrees of freedom were adjusted using the Huynh-Feldt correction, and were reported accordingly. Effect sizes were calculated as partial eta squared values $\left(\eta_{\mathrm{p}}^{2}\right)$.

\section{Results}

\section{Trial Duration}

We found no significant effects of trial duration: across trials, $M=1.46, S D=0.12$. 


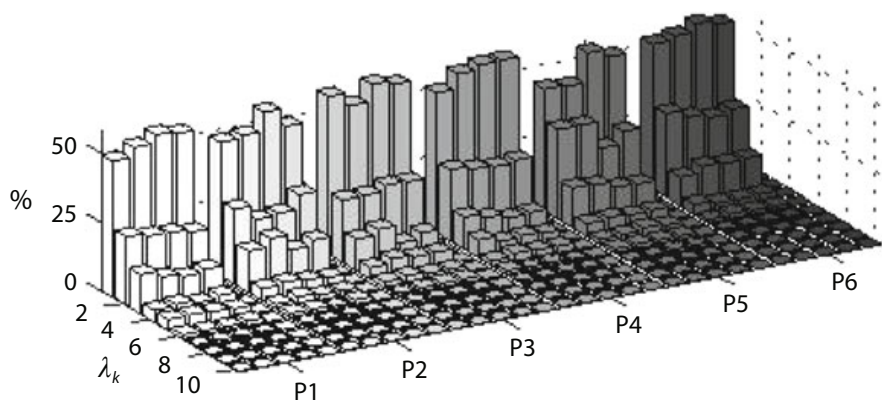

Figure 2. Eigenvalue spectra for the first $\mathbf{1 0}$ modes for each condition for all 6 participants (P1 . . . P6). For each participant, the first, second, third, and fourth rows represent the inside-out short/deep and cross-court short/deep conditions, respectively.

\section{Time Series Standard Deviation $\left(T S_{S D}\right)$}

There was a significant main effect of shot distance $\left[F(1,5)=6.75, p<.05, \eta_{\mathrm{p}}^{2}=.57\right]$; the $T S_{S D}$ of the deep shots exceeded that of the short shots $(M=4.94 \mathrm{~mm}$, $S D=4.22$, and $M=4.87 \mathrm{~mm}, S D=4.22$, for deep and short shots, respectively). In addition, there was a significant effect of shot direction $\left[F(1,5)=47.98, p<.01, \eta_{\mathrm{p}}^{2}=\right.$ $.90]$, and the $T S_{S D}$ was larger for the cross-court shots than for the inside-out shots $(M=5.02 \mathrm{~mm}, S D=4.38$ vs. $M=4.79 \mathrm{~mm}, S D=4.05$ ).

\section{PCA}

Shot comparisons across participants. The PCA conducted on the four trials for each condition per participant revealed that the first three modes captured roughly $90 \%$ of all the variance in each 216-dimensional data set. The corresponding eigenvalue spectra were very similar (see Figure 2).
For each shot condition, the covariance between the projections $\xi_{k}(t)$ corresponding to each participant for the first three modes hinted at a high degree of similarity among participants for each condition. The covariance between the projections was sometimes negative in some participants. In these cases, the sign of most corresponding eigenvector coefficients differed, indicating that the trajectories of the majority of the corresponding time series were very similar. We therefore report the absolute value of the covariance. The mean absolute value of the covariance across participants was close to 1 , and the standard deviations were low (see Table 1). Furthermore, the degree of similarity of the projections of the different conditions was similarly high; the mean absolute value of the covariance across participants between the projections of the different shots (i.e., inside-out short/deep, cross-court short/deep) was close to 1 , and the corresponding standard deviations were close to 0 (see Table 2). These results indicated a high

Table 1

Means and Standard Deviations of the Absolute Values of the Covariance Between the Corresponding Projections $\xi_{k}(t)$ of Shots, Across Participants for the First Three Modes

\begin{tabular}{|c|c|c|c|c|c|c|}
\hline & \multicolumn{2}{|c|}{ Mode 1} & \multicolumn{2}{|c|}{ Mode 2} & \multicolumn{2}{|c|}{ Mode 3} \\
\hline & $M$ & $S D$ & $M$ & $S D$ & $M$ & $S D$ \\
\hline IOS & .97 & .03 & .94 & .04 & .93 & .03 \\
\hline IOD & .96 & .04 & .91 & .05 & .94 & .03 \\
\hline $\mathrm{CCS}$ & .94 & .06 & .91 & .06 & .93 & .04 \\
\hline CCD & .97 & .03 & .95 & .03 & .96 & .02 \\
\hline
\end{tabular}

Note-Each entry corresponds to the 15 pairwise comparisons between the 6 participants.

Table 2

Means and Standard Deviations of the Absolute Values of the Covariance Between the Projections $\xi_{k}(t)$ of the Short (S) and Deep (D) Inside-Out (IO) and Cross-Court (CC) Shots, Across Participants for the First Three Modes

\begin{tabular}{|c|c|c|c|c|c|c|c|c|c|c|c|c|c|c|c|c|c|c|}
\hline & \multicolumn{6}{|c|}{ Mode 1} & \multicolumn{6}{|c|}{ Mode 2} & \multicolumn{6}{|c|}{ Mode 3} \\
\hline & \multicolumn{2}{|c|}{ IOD } & \multicolumn{2}{|c|}{$\mathrm{CCS}$} & \multicolumn{2}{|c|}{$\mathrm{CCD}$} & \multicolumn{2}{|c|}{ IOD } & \multicolumn{2}{|c|}{$\mathrm{CCS}$} & \multicolumn{2}{|c|}{$\mathrm{CCD}$} & \multicolumn{2}{|c|}{ IOD } & \multicolumn{2}{|c|}{$\mathrm{CCS}$} & \multicolumn{2}{|c|}{$\mathrm{CCD}$} \\
\hline & $M$ & $S D$ & $M$ & $S D$ & $M$ & $S D$ & $M$ & $S D$ & $M$ & $S D$ & $M$ & $S D$ & $M$ & $S D$ & $M$ & $S D$ & $M$ & $S D$ \\
\hline IOS & .99 & .02 & .97 & .03 & .99 & .01 & .97 & .02 & .96 & .03 & .99 & .01 & .97 & .01 & .97 & .02 & .95 & .04 \\
\hline IOD & & & .98 & .02 & .99 & .01 & & & .96 & .02 & .98 & .01 & & & .96 & .02 & .98 & .01 \\
\hline $\mathrm{CCS}$ & & & & & .98 & .02 & & & & & .96 & .02 & & & & & .96 & .02 \\
\hline
\end{tabular}

Note-Each entry corresponds to six pairwise comparisons. 
degree of similarity among the shots at the level of the most prominent modes. Potential differences between shot conditions should therefore reside in a differential contribution from the anatomical landmarks and/or racket. Consequently, instead of analyzing the corresponding eigenvectors, we conducted a separate PCA in which the time series of all conditions and participants were included.

General observations. PCA showed that the entire data set could effectively be described by a few modes; the cumulative sum of the first five eigenvalues $\lambda_{k}$ represented $52.6 \%, 77.6 \%, 89.3 \%, 93.6 \%$, and $96.2 \%$ of all the variance in the data set, respectively. The corresponding projections [i.e., time evolutions, $\xi_{k}(t)$; see Equation 3] are shown in Figure 3.

To determine the contribution to specific modes from the various marker locations, we calculated the mean absolute value of the eigenvector coefficients $v_{i}^{k}$ for $k=1 \ldots 5$ (see Equation 1) across participants and conditions. Similarly, to address the variability of these contributions, we calculated the standard deviation of the signed value of the eigenvector coefficients. The results of these analyses are depicted in Figures 4 and 5, respectively. The differences in magnitude of the eigenvector coefficients were small in the first mode (at least for the $x$ - and $y$-directions) and became more pronounced in the higher modes. In particular, anatomically localized and Cartesian direction-specific contributions were found in the dominant modes, although less so in the first. In addition, whereas the contribution of the shoulder-armracket linkage was substantial for the first three modes, this contribution was markedly smaller for the fourth and fifth modes. In general, the mean magnitude of the eigenvector coefficients across marker locations decreased monotonically from Modes 1 to 5, and their averaged standard deviation increased. In other words, the variance (from different marker locations) within modes was distributed less homogeneously for increasingly higher modes.

Comparisons among shots. In view of the large number of statistical analyses undertaken $(18$ marker locations $\times 3$ movement directions $\times 5$ modes $=216$ ANOVAs), the re-

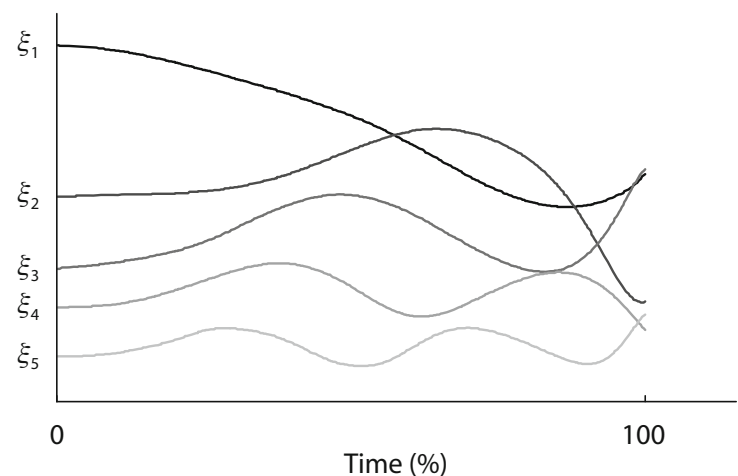

Figure 3. Projections $\left(\xi_{k}\right)$ corresponding to the first 5 modes of the PCA when all trials were included. Time is indicated on the horizontal axis as a percentage of the entire action (see text). The position on the vertical axis has been shifted for visualization purposes. sulting values are reported in the Appendix in Tables A1 and A2. Marker locations for which a significant effect for shot direction was found are indicated for Modes 1 to 5 in Figure 6, which represents only variations in shot direction, because few significant differences in shot distance were observed. More specifically, significant differences in shot direction versus distance were found in 13 versus 0,11 versus 3,10 versus 1,4 versus 3 , and 12 versus 2 analyses, for Modes 1,2,3,4, and 5, respectively. Below, we discuss only significant differences in shot direction.

As can be seen in Figure 6, characteristic differences between shot directions (in terms of dynamic structures and Cartesian directions) across participants were repeatedly found in terms of differential eigenvector coefficients. The observed differences were predominantly, but not exclusively, present in the $x$-direction (i.e., the mediolateral direction). Although these differences were predominantly found on the right side of the body - specifically in the shoulder-racket linkage - across the first five modes and directions, significant differences in eigenvector coefficients occurred all over the body and racket.

\section{Discussion}

We examined different types of tennis shots in terms of their dynamic modes, as well as their scaling properties (as quantified by the standard deviations of the time series), using PCA. We found that five principal modes captured more than $96 \%$ of the variance in the data, which was distributed unevenly across the body and racket. Furthermore, the variability of corresponding eigenvector coefficients was unevenly distributed across the different body areas and the racket, and was larger for the higher modes. These results indicate that tennis shots can be given a compact description and suggest that their execution may be governed by a low-dimensional control structure. Although this finding is not necessarily new (see, e.g., Huys, Daffertshofer, \& Beek, 2004; Post, Daffertshofer, \& Beek, 2000 ), it has, as far as we know, never been reported for a whole-body, complex discrete task.

As expected, neither shot direction nor shot depth was characterized by a unique structure. However, clear shot-direction differences and, to a much lesser extent, shot-distance differences were evident in the eigenvector coefficients of the various modes. Such differences occurred across modes all over the body and the racket; they were most pronounced in the $x$-direction, particularly at the right side of the body and the racket (for a representation of differences in shot direction across the first three modes, see Figure 8). The latter observation is consistent with previous research that suggests that racket, racketholding arm, shoulders, and hips are important for anticipation, albeit in a skill-dependent fashion (see Ward et al., 2002; Williams et al., 2002). The present results indicate that the information contained in those regions is largely confined to the $x$-direction. In addition, we found shotdirection-specific differences in the hips and shoulders in all directions and in anatomical locations that were not identified as potentially informative regions in previous studies, such as the left arm and right leg (in the $y$ - and 

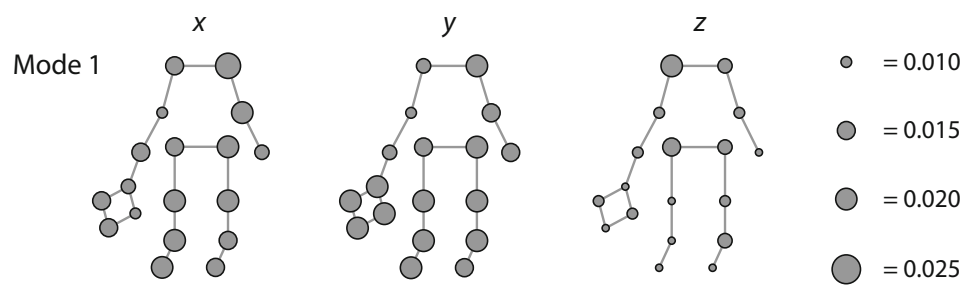

Mode 2
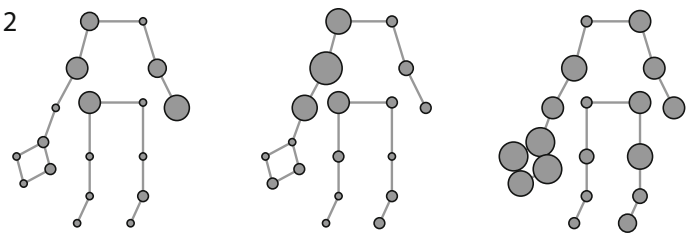

Mode 3
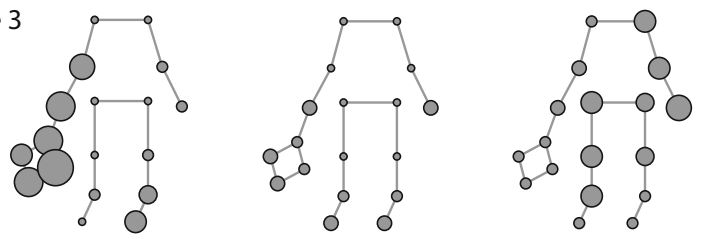

Mode 4
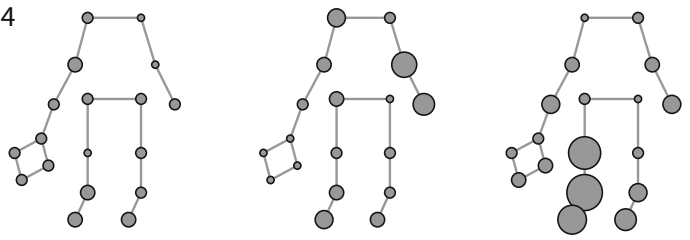

Mode 5
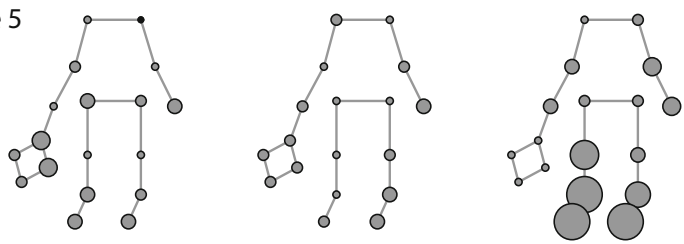

Figure 4. Stick figures indicating the mean across participants and trials of the absolute magnitude of the eigenvector coefficients at each anatomical location for Modes 1 to 5 in the $x$-, $y$-, and $z$-directions (left, middle, and right columns, respectively) (see text). The marker size corresponds to the absolute magnitude.

$z$-directions). The scaling of shot dynamics, quantified by the $T S_{S D} \mathrm{~S}$, distinguished the inside-out shots from the cross-court shots, as well as the short from the deep shots. That is, differences in shot direction were found in the shot dynamics, as well as in the scaling. In contrast, differences in shot depth were rarely apparent in the shot dynamics as such, but its scaling was larger in the deep shots than in the short shots. Shot depth, at least in the present context, appears to be controlled primarily by a scaling of the dynamics and less so by coordinative dynamic adjustments in shot execution. It should be noted, however, that the differential effect on the dynamics of shot direction and distance may be due to differences in their respective target discrimination, even though the scaling results contradict this suggestion. Following our premise that anticipation is based on dynamic information, these results suggest that the direction of tennis passing shots is easier to anticipate than their depth.

In sum, a similar low-dimensional dynamic structure captures the execution of tennis passing shots to distinct directions and of different depths. Differences between shots arose locally (i.e., they were found in varying contributions of different body and racket locations to these dynamics), but mainly so for shot direction. In contrast, the scaling of the dynamics was distinct for both shot direction and distance.

\section{EXPERIMENT 2}

In Experiment 1, we found that a few modes allowed for an economical description of forehand tennis shots, and that subtle differences in these modes could distinguish shot direction, but not shot depth. However, any description 
Mode 1

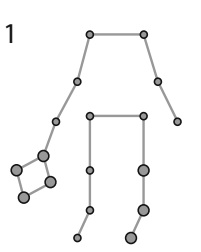

Mode 2

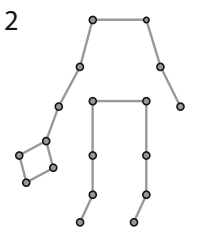

Mode 3

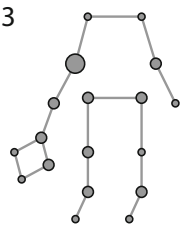

Mode 4

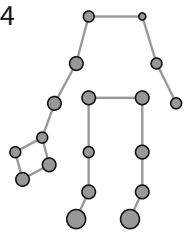

Mode 5

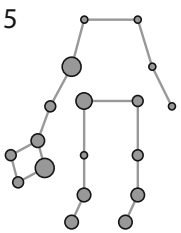

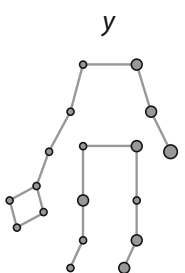

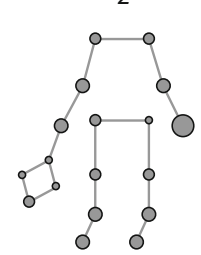

$\circ=0.010$

$\bigcirc=0.015$

$O=0.020$

$=0.025$
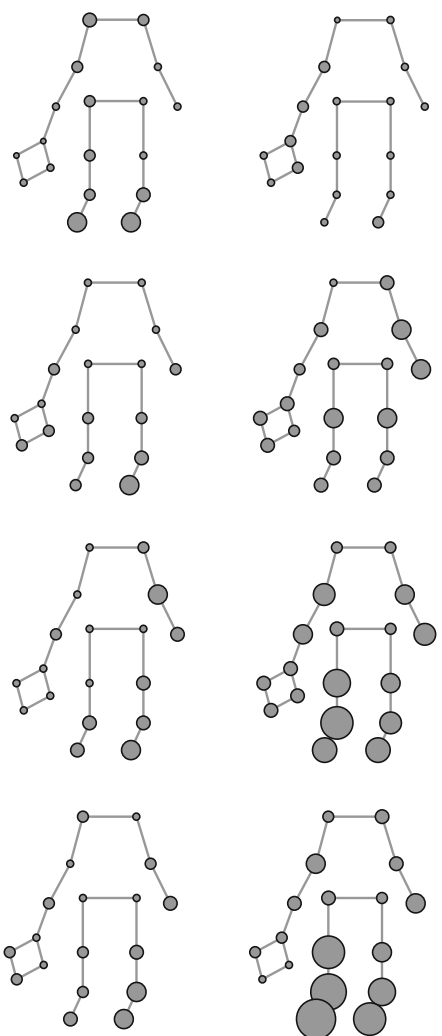

Figure 5. Stick figures indicating the standard deviations across participants and trials of the real value of the eigenvector coefficients at each anatomical location for Modes 1 to 5 in the $x$-, $y$-, and $z$-directions (left, middle, and right columns, respectively) (see text). The marker size corresponds to the absolute magnitude.

of a system in fewer dimensions than the original represents by definition an approximation. Therefore, we conducted a second experiment aimed at identifying the minimal dimensionality of forehand tennis shots allowing for undisrupted anticipation of shot direction (i.e., with an accuracy level similar to that of "real" shots). In other words, we sought to find the most efficient representation of the shots from a perceptual perspective. Moreover, we wanted to examine whether anticipation skill interacted with the perceptually most efficient representation of the shots. To achieve this aim, we simulated tennis shots that were constructed from the original data, as well as shots that were constructed by cumulatively including five principal modes. Subsequently, skilled and less skilled tennis players were requested to observe the simulations and judge shot direction. In contrast with other researchers (e.g., Ward et al., 2002; Williams et al., 2002), we examined anticipation performance only in terms of accuracy because we wanted participants to look at the entire simulation (rather than allowing them to control viewing time), thereby eliminating a potential confound.

We expected anticipation accuracy not to be affected when most of the variance of the shots was incorporated in the simulations - that is, when minimally the first three modes (accounting for approximately $90 \%$ of the variance) were included. Moreover, since the addition of more modes barely changed the shot dynamics (they accounted for only a small amount of the variance), we expected anticipation performance to improve only marginally at best, and only in skilled participants, because they are able to pick up and use subtle cues (Shim et al., 2005; Ward et al., 2002). Generally, we expected skilled participants to demonstrate performance superior to that of their less skilled counterparts. 
$X$

Mode 1

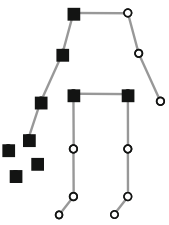

Mode 2

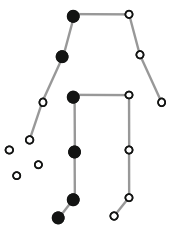

Mode 3

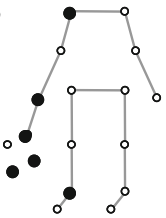

Mode 4

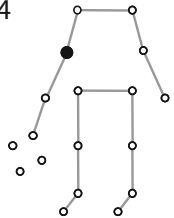

Mode 5

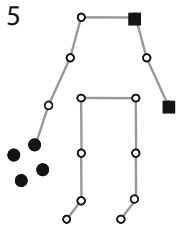

$y$
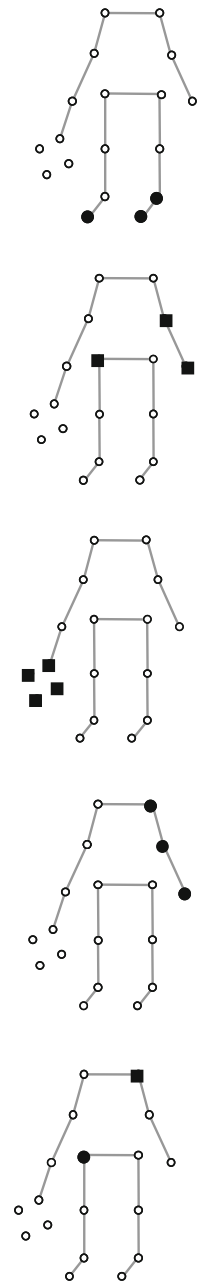

$z$
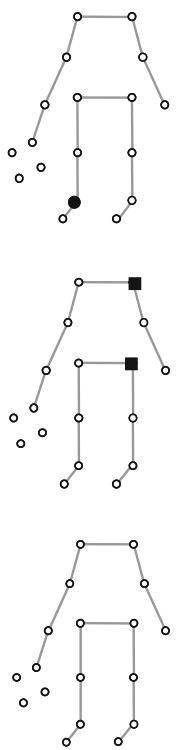

Figure 6. Stick figures representing the results of the statistical analysis for shot direction (see text). Black markers represent locations for which a significant difference between shot directions was found at the $\alpha=.05$ level. Circles indicate that the absolute value of the mean eigenvector coefficients of the inside-out shot was larger than that of the crosscourt shot, whereas the reverse is the case for the squares. Rows 1 to 5 represent the results for Modes 1 to 5; the left, middle, and right columns represent the results for the $x$-, $y$-, and $z$-directions, respectively.

\section{Participants}

Twelve moderately skilled participants (mean age $=34.0$ years, $S D=11.6$ ) who had played regular tennis at school (albeit at a recreational level), had never received professional tennis coaching, and did not play in tennis tournaments, and 13 highly skilled participants (mean age $=26.6$ years, $S D=11.1$ ) who all competed at a national level (mean LTA rating $=4.78, S D=2.67$ ) and played, on average, 134 tournament games per annum, volunteered to participate.

\section{Apparatus and Stimuli}

Stick-figure simulations of tennis shots were generated using MATLAB (version 6.5, The MathWorks). The simulations were based on a single inside-out and cross-court shot from two partici- pants and were saved in audio video interleave (.avi) format. These shots were selected because the corresponding eigenvectors of the first three modes most closely resembled the shot-specific mean eigenvectors (as determined in Experiment 1) of those modes, in terms of least mean squares. In contrast, using shot deliveries from a single player would run the risk of contaminating anticipation on the "original" shots by introducing player-specific motion patterns; using multiple players would inevitably result in simulations with larger deviations from the shot-specific mean eigenvector in terms of least mean squares. Simulations of shots in both directions were made from original data $\left(\mathrm{Mode}_{C}\right)$ and from data containing Modes 1 to 5 via cumulative addition of these modes, referred to as $M_{0} e_{1}$, Mode $_{1-2}$, Mode $_{1-3}$, Mode $_{1-4}$, and Mode ${ }_{1-5}$, respectively. For the latter five conditions, data $\mathbf{q}(t)$ for the simulations were generated by 
computing the product of the time evolutions and the corresponding eigenvectors of these modes [i.e., $\mathbf{q}(t)=\xi_{k}(t) \cdot v_{i}^{k}$, for $k=1 \ldots 5$, and $i=1 \ldots 54$; see also Equation 1]. Thus, for each condition, a 54-dimensional vector $\mathbf{q}(t)$ was obtained that represented the dynamics corresponding to the 18 marker locations in three Cartesian directions. The data's "real-world" coordinates were then obtained by multiplying each (marker's) time series $q_{i}(t)$ with its corresponding marker-specific mean standard deviation (averaged across shot directions) and adding its original mean. To clarify, the so-obtained stick figures were structurally the same as that of the original shots, whereas the dynamic differences relative to the original shots were larger the fewer modes were included. This procedure resulted in 12 experimental conditions ( 6 modes $\times 2$ players). The frame rate for the simulations was $30 \mathrm{~Hz}$. We resampled the corresponding time series of the selected shots to a multiple of 30 , and minimized changes in the number of samples. Note that the time series that were used for the simulations were first normalized to unit variance and subsequently rescaled according to the corresponding mean standard deviation across shot directions in order to eliminate any possible information in the simulations with regard to shot direction, due to scaling. The stick figures were presented in black against a white background.

The clips were imported into Adobe Premier 6.0 on a notebook computer (Sony) with a 15 -in. screen. The size of the simulated players was approximately $12 \times 14 \mathrm{~cm}$ (height $\times$ width). The trials were edited so that they were preceded by a $1-\mathrm{sec}$ presentation of a white background with a centrally placed black dot, followed by a 1 -sec presentation of the white background alone. Subsequently, the simulated shot was presented for approximately $1.5 \mathrm{sec}$. After presentation, a white background was shown for $3 \mathrm{sec}$, during which the participants had to indicate verbally whether the presentation shown entailed a shot to their left side (i.e., inside-out) or to their right side (i.e., cross-court). The trials were randomized and presented in four blocks of 30 trials. A practice test tape of 20 trials, in which original $\left(\right.$ Mode $\left._{C}\right)$ inside-out and cross-court shots were presented 10 times in a blocked fashion, was constructed in a similar manner.

\section{Procedure}

From a distance of about $0.5 \mathrm{~m}$ while seated, participants viewed the laptop on which the trials were presented. The center of the display was approximately at eye height. Participants were told that they would be shown tennis shots in which the ball was played either inside-out or cross-court. They were told to imagine themselves being located at the center of a tennis court at the middle of the baseline and that the to-be-anticipated shots were delivered from the same position on the opponent's side of the court. They were also told that the shots were to be delivered by a headless stick figure, that the clips would last up to the moment of ball-racket contact, and that no ball would be presented. Participants were notified that each trial would be preceded by a white screen onto which a small black dot would be projected and visible for $1 \mathrm{sec}$, after which the screen would be totally white for $1 \mathrm{sec}$, followed by the simulation. Participants were requested to verbally indicate the direction of each shot (left or right) after the simulation was finished. Each simulation was presented five times, resulting in a total of 120 trials. Before the experiment proper, participants were shown 10 examples of original $\left(M_{o d e}\right)$ shots to each direction in blocks of five shots. The direction of these shots was indicated before their presentation. The simulations were presented in blocks of 30 trials, followed by a short break. The entire experiment lasted about $20 \mathrm{~min}$.

\section{Data Analysis}

We determined the number of correct answers $(c)$ for each experimental condition. In order to minimize the corresponding distribution's deviation from normality, we subsequently transformed this number using Bartlett's modified arcsine transformation - that is,

$$
p^{\prime}=(360 / 2 \pi) \arcsin \sqrt{(c+3 / 8) /(n+3 / 4)},
$$

with $n$ being the number of trials (Bartlett, 1937, as cited in Zar, 1996). These scores were analyzed using a mixed design ANOVA with player and mode as within-participants factors and skill as a between-participants factor. We used a contrast analysis to follow up any significant main effect of mode (the only main effect with more than two levels). We report and graph the untransformed means and standard deviations that were calculated from the original data.

\section{Results}

The accuracy of anticipating the original shots was similar to that reported in previous studies (approximately $75 \%$ across skill groups; see Ward et al., 2002; Williams et al., 2002), which validates our stick figure simulations. The anticipation of shots delivered by Players 1 and 2 did not differ significantly $\left[F(1,24)=0.002, p=.961, \eta_{\mathrm{p}}^{2}<\right.$ $.001]$. There was, however, a significant player $\times$ mode interaction $\left[F(5,120)=8.374, p<.001, \eta_{\mathrm{p}}^{2}=.259\right]$, which revealed that the shot delivery of the original $\left(\operatorname{Mode}_{C}\right)$ presentation of Player 1 was anticipated better than that of Player 2, whereas the reverse was the case when the first four modes $\left(\operatorname{Mode}_{1-4}\right)$ were included in the simulation.

As expected, a significant group effect $[F(1,24)=$ $14.272, p<.005, \eta_{\mathrm{p}}^{2}=.373$ ] was observed; the skilled players anticipated more accurately than their less skilled counterparts did $(M=77.1 \%, S D=15.6$ vs. $M=66.6 \%$, $S D=14.2$, respectively). There was also a significant effect of mode $[F(4.651,111.621)=31.403, p<.001$, $\left.\eta_{\mathrm{p}}^{2}=.567\right]$ (for Mode $_{C}, M=76.9 \%, S D=13.0$; for Mode $_{1}, M=56.7 \%, S D=15.9$; for Mode $_{1-2}, M=61.0 \%$, $S D=9.4$; for Mode $_{1-3}, M=80.2 \%, S D=13.1$; for Mode $_{1-4}$, $M=78.1, S D=9.1$; for Mode $_{1-5}, M=80.6, S D=14.9$ ). Contrast analysis indicated that the presentations of shots containing Mode $1\left(\right.$ Mode $\left._{1}\right)$ and Modes 1 and $2\left(\right.$ Mode $\left._{1-2}\right)$ were anticipated less accurately than were the presentations of the original $\left(\mathrm{Mode}_{C}\right)$ shots. In addition, there was a tendency for the shots containing Modes 1 to 5 (Mode $\left.{ }_{1-5}\right)$ to be anticipated more accurately than the original shots $(p=$ .051). The group $\times$ mode interaction just failed to reach significance $\left[F(4.651,111.621)=2.240, p=.060, \eta_{\mathrm{p}}^{2}=\right.$ $.085]$, although only the less skilled tended to perform bet-

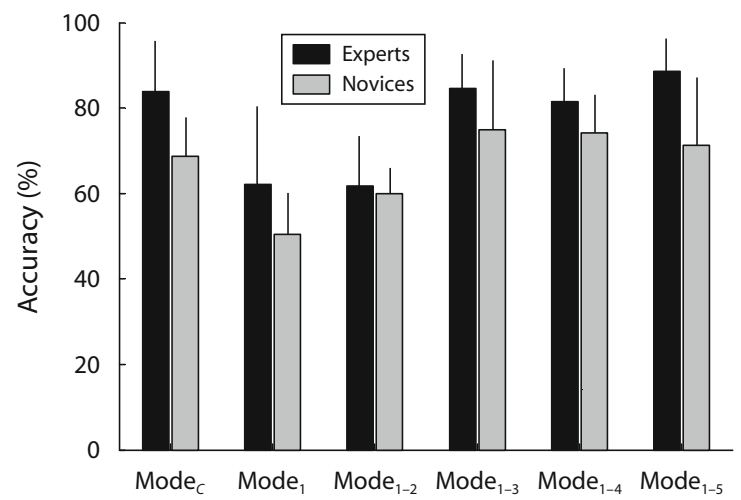

Figure 7. Response accuracy as a function of skill level and information content (i.e., the number of modes included in the simulations). 
ter when three, four, or five modes were included than when only the original shots were presented (see Figure 7).

Visual inspection of the data suggested that when we presented only Mode $1\left(\right.$ Mode $\left._{1}\right)$, the less skilled performed at chance level, in contrast with the skilled players. Therefore, we performed a one-sample $t$ test for each groupmode combination to examine whether performance was significantly above chance (i.e., 50\% correct). These analyses confirmed our impression that when only Mode 1 was presented, the less skilled performed at chance; in all other cases, performance was significantly better than chance $(\alpha=.05)$.

\section{Discussion}

We examined the ability of skilled and less skilled tennis players to anticipate simulated shots whose dynamics either were unmodified (i.e., original shots) or were constructed by cumulatively including Modes 1 to 5 as a function of skill. The shots performed by two players from Experiment 1 served as data for the simulations.

As expected, anticipation was, on average, unaffected by the player factor. The significant player $\times$ mode interaction, however, indicated that anticipation performance of the original shot was player dependent, which may be readily explained in terms of idiosyncrasies in technique. Also, anticipation of shots delivered by Player 1, when including the first four modes, was worse than that for shots delivered by Player 2. In order to examine this effect, we further analyzed the eigenvectors corresponding to the fourth and fifth modes in terms of their least squares differences from the mean eigenvector (see also the Method section). It appeared that for Mode 4, the least squares difference of the shot delivered by Player 1 was larger than that of Player 2, which may explain the decreased response accuracy in the corresponding condition. In our opinion, this small deviation did not interfere with the primary aim of the experiment, which was to find the extent to which the information content of the simulations could be reduced without affecting anticipation performance relative to original shots.

With regard to this objective, we found strong evidence that simulating shot deliveries based on the first mode $\left(\right.$ Mode $\left._{1}\right)$ and first two modes $\left(\right.$ Mode $\left._{1-2}\right)$ negatively affected anticipation performance, but simulations created by including the first three, four, or five modes (corresponding to approximately $90 \%$ of the variance in the shot deliveries, or more) had no detrimental effect on performance. There are two important implications of these results. First, the information for judging shot direction resides in or is condensed in a limited number of orthogonal (i.e., independent) structures or modes, and is therefore identifiable. This finding indicates that a lowdimensional, compact representation of tennis shots is not only optimal from a mathematical point of view, but is also most informative in terms of perception. Second, the results of Experiment 1 indicated that across the first three modes, significant dynamic differences between shot directions occurred at almost all body areas, as well as the racket. That is, information regarding shot direction may, in principle, be gleaned from all parts of the body. Furthermore, anticipation performance tended to be better, albeit only slightly, when Modes 1 to $5\left(M_{\text {ode }}{ }_{1-5}\right)$ were simulated than when the original shots were presented. This tendency suggests that the information contained in the higher modes (as present in the original shots) may negatively affect anticipation performance relative to the apparent information-optimal representation (i.e., Mode $_{1-5}$ ), at least in the present context, probably because the additional variance (information) is primarily nonspecific to shot direction, representing, for instance, trial-to-trial variability, thus hampering rather than improving anticipation performance. Further research is required for examining this issue in more detail.

In line with previous research, the skilled players were more accurate in their anticipation judgments than were their less skilled counterparts (see, e.g., Abernethy, 1990; Shim et al., 2005; Williams et al., 2002). In addition, we found evidence that the skilled players, unlike the less skilled participants, were able to use the information presented in Mode 1, and that the novices tended to respond more accurately when Modes 3, 4, and 5 (Mode $_{1-3}$, Mode $_{1-4}$, Mode $_{1-5}$ ) were present than when only the original $\left(\right.$ Mode $\left._{C}\right)$ shots were presented. These findings suggest that experts are able to pick up and use dynamic information of a lower dimension than less skilled players can, and that the performance of only the latter deteriorates when information that is largely nonspecific to shot direction is present. The skilled players appear to have learned to perceptually identify the dynamic invariants that indicate shot direction to a greater degree than less skilled participants, and are therefore less vulnerable to random variability across shot executions. Furthermore, the performance of less skilled players can be optimized by presenting them with low- (i.e., three-) dimensional dynamic information, a finding that may have important consequences for training perceptual skill.

In sum, information that facilitates the anticipation of shot direction is in large part dynamic and resides in a few independent structures. Skilled players are able to identify and use these informational dynamics to a greater extent than less skilled participants can. The ability to pick up low-dimensional information may render perception reliable and robust.

\section{EXPERIMENT 3}

In Experiment 2, we examined whether low-dimensional dynamic information allows for anticipating shot direction. We did this by presenting skilled and less skilled tennis players with simulated shots that we had generated by combining the most important modes identified in Experiment 1 . We found that only three structures (modes) contained all the information necessary for anticipation at a level similar to that observed for the original shots. Unfortunately, the methodology adopted did not allow us to identify exactly which dynamic structures were used for anticipation. Moreover, increasing the number of structures for the simulations increased the variance accounted for vis-à-vis shot representation. In the present experiment, we attempted to pinpoint the dynamic structures that allow for anticipation of shot direction as a function of skill level. We generated stimuli in which only one or 
a combination of structures contained information about shot direction, and information in the remaining structures was eliminated. The amount of variance of the shot simulations (i.e., representations) was, therefore, kept nearly constant. In addition to a control condition, in which the unmodified mean eigenvectors were used (see the Method section), we generated simulations in which the eigenvectors corresponding to the following modes remained unmodified (i.e., contained shot directional differences): Modes 1 to 5 presented separately, the combination of Modes 1 and 2, Modes 1 and 3, Modes 2 and 3, Modes 6 and 7, and Modes 8 to 20. The latter two conditions were included to examine whether the higher modes contained information that could be used at all by observers. We hypothesized that the more variance a mode or combination of modes contained, the more it would allow for anticipation performance. Furthermore, although we did not expect any single mode to allow for anticipation at the level observed for the control condition, we expected the first three modes individually to allow for anticipation above chance level - at least for the experts - because the results of Experiment 1 had revealed that these modes contained quite some variance and clearly revealed shot-directionspecific differences in terms of the eigenvectors. We also expected that, regardless of skill level, the combination of Modes 1 and 3 would contain sufficient information to allow for undistorted anticipation performance, because in Experiment 2, the inclusion of Modes 1 to 3 had allowed for accurate performance, whereas adding Mode 2 to Mode 1 had barely affected performance.

Pilot work indicated that presenting all the conditions to participants was too strenuous. The tested participants complained about fatigue, fading attention, and flagging motivation during the test, even though breaks were provided. Therefore, we decided to split the experiment into Parts A and B.

\section{Method}

\section{Participants}

Fourteen less skilled participants (mean age $=39.9$ years, $S D=$ 11.7) who had played tennis recreationally at school, had never received any professional tennis coaching, and did not play in tennis tournaments, and 14 skilled participants (mean age $=22.1$ years, $S D=4.5$ ) who had competed at a national level (mean LTA rating $=2.98, S D=1.65)$ and played an average of 175 games annually $(S D=43)$, volunteered to participate in Part A. In Part B, 14 less skilled participants (mean age $=38.3$ years, $S D=10.8$ ) who had played tennis recreationally at school, had never received any professional tennis coaching, and did not play in tennis tournaments, and 14 skilled participants (mean age $=26.8$ years, $S D=$ 11.4) who competed at a national level (mean LTA rating $=3.40$, $S D=2.18)$ and played an average of 153 games annually $(S D=69)$, volunteered to participate.

\section{Apparatus and Stimuli}

The simulations were generated in a manner similar to those in Experiment 2. However, in contrast with Experiment 2, the data for the simulations were based on the mean eigenvectors, as determined in Experiment 1-that is, for each coefficient $v_{i}^{k}(k=1 \ldots 54 ; i=$ 1 ...54), the means across participants and trials for each shot direction were computed. Differences in eigenvector coefficients in shot distance were averaged out for each mode. For the time series corresponding to the marker locations of each participant, we calculated the mean and standard deviation across shot distance and direction. These means and standard deviations were used to obtain data with "real" spatial properties and simultaneously to eliminate the potential impact on anticipation of the shots' spatial properties (see Experiment 1). For the simulations, we selected the means and standard deviations of the 2 participants who had the smallest sum of squares between the across-participant averages of the $T S_{S D} \mathrm{~S}$ and their own. In all of the simulations, 54 modes (capturing more than $99 \%$ of the entire variance in the data set) were used. For each shot direction, simulations were made of original shots, in which the shot differences that were present in the eigenvectors were preserved, and shots in which only a single mode or a combination of modes maintained shot differences (as present in the corresponding eigenvectors), and shot differences in all other modes were averaged out. Specifically, in constructing the data as $\mathbf{q}(t)=\xi_{k}(t) \cdot v_{i}^{k}, k=1 \ldots 54$, and $i=1 \ldots 54$ (see Equation 1), we used $v_{i}^{k}=v_{(\mathrm{IO}) i} i$ and $v_{(\mathrm{XC}) i}$ for the modes in which shot differences were preserved, and shot differences were averaged out using $v_{i}^{k}=\left(v_{(\mathrm{IO}) i}^{k}+v_{(\mathrm{XC}) i}{ }^{k}\right) / 2$ (for $\left.i=1 \ldots 54\right)$ for the other modes (the subscripts IO and XC refer to the inside-out and cross-court shots, respectively). The same procedure as was used in Experiment 2 was used to obtain data with "real-world" coordinates and to ensure that the stick figures were structurally the same as for the original shots. The following modes were chosen: Modes $1\left(M_{1} d_{1}\right), 2\left(M_{0} e_{2}\right)$, 3 (Mode $\left._{3}\right), 4\left(\right.$ Mode $\left._{4}\right)$, and $5\left(\right.$ Mode $\left._{5}\right)$; Modes 1 and $2\left(\right.$ Mode $\left._{1+2}\right)$; Modes 1 and 3 (Mode $1+3)$; Modes 2 and 3 (Mode $2+3$ ); Modes 6 and 7 (Mode $\left.{ }_{6+7}\right)$; and Modes 8 to $20\left(\right.$ Mode $\left._{8-20}\right)$. For the last condition, our motivation for going up to Mode 20, instead of to a lower or higher mode, was that in this manner the amount of variance accounted for in this condition was almost equal to that of the combination of Modes 6 and $7\left(\operatorname{Mode}_{6+7}\right)$. For instance, in condition $\mathrm{Mode}_{3}$, as always, all 54 modes were used, but only the shot-direction-specific mean of the eigenvector coefficients corresponding to Mode 3 were used without alteration; the eigenvector coefficients of the other modes (i.e., Modes 1 and 2 and Modes 4-54) were averaged across shot direction and thus contained no shot differences (and, therefore, no information that could be used for anticipation). The conditions $M_{0} e_{C}$, Mode $_{2}$, Mode $_{5}$, Mode $_{1+2}$, Mode $_{1+3}$, and Mode ${ }_{2+3}$ were used in Part A, whereas the conditions Mode, Mode 1, Mode $_{3}$, Mode $_{4}$, Mode $_{6+7}$, and Mode $_{8-20}$ were used in Part B.

\section{Procedure}

The procedure was the same as that used in Experiment 2.

\section{Data Analysis}

As in Experiment 2, we determined the percentage of correct answers for each experimental condition and subjected these percentages to Bartlett's modified arcsine transformation (Bartlett, 1937, as cited in Zar, 1996). These scores were analyzed using a mixed design ANOVA with player and mode as within-participants factors and skill as a between-participants factor. We used Bonferroni-corrected pairwise comparisons to locate the origin(s) of a potential significant main effect of mode.

\section{Results}

\section{Part A}

We found a significant effect of player $[F(1,26)=$ $\left.15.652, p=.001, \eta_{\mathrm{p}}^{2}=.376\right]$, which indicated that the response accuracy was higher for Player 1 than for Player 2 $(M=65.8 \%, S D=15.6$ vs. $M=60.0 \%, S D=16.5$, for Players 1 and 2, respectively). Unexpectedly, no significant effect of skill was found, even though on average the skilled players $(M=64.4 \%, S D=16.1)$ responded more accurately than did the less skilled players $(M=61.4 \%$, $S D=16.4)$. We find it important that there was a significant effect of mode $\left[F(5,130)=6.208, p=.000, \eta_{\mathrm{p}}^{2}=\right.$ .193] (for Mode $C, M=70.2 \%, S D=19.9$; for $M_{0} e_{2}$, 
$M=61.8 \%, S D=11.2 ;$ for Mode $_{5}, M=57.3 \%, S D=10.8$; for Mode $_{1+2}, M=60.2 \%, S D=13.4$; for $M o d e_{1+3}$, $M=63.9 \%, S D=11.7$; for Mode $_{2+3}, M=64.1 \%$, $S D=13.0)$. The pairwise comparisons showed that anticipation accuracy in the control condition $\left(\right.$ Mode $\left._{C}\right)$, the conditions with Modes 1 and $3\left(\right.$ Mode $\left._{1+3}\right)$, and those with Modes 2 and $3\left(\right.$ Mode $\left._{2+3}\right)$ did not differ significantly, and that a tendency for a lack of differences was present vis-àvis Mode $2\left(\right.$ Mode $\left._{2}\right)$. The accuracy in these three conditions differed significantly from that in the other conditions (i.e., Mode $_{5}$, Mode $\left._{1+2}\right)$, which, in turn, did not significantly differ from each other. Furthermore, anticipation accuracy in each condition (i.e., Mode $_{C}$, Mode $_{2}$, Mode $_{5}$, Mode $_{1+2}$, Mode $_{1+3}$, Mode $_{2+3}$ ) was above chance (at $\alpha=.05$ ).

\section{Part B}

As in Part A, there was a main effect of player $[F(1,24)=$ $\left.6.612, p=.017, \eta_{\mathrm{p}}^{2}=.216\right](M=65.5 \%, S D=17.5 \mathrm{vs}$. $M=59.3 \%, S D=16.3$, for Players 1 and 2, respectively). The effect of skill did not reach significance, although the skilled group $(M=63.5 \%, S D=17.2)$ responded more accurately than did less skilled players $(M=61.4 \%$, $S D=17.1)$. The effect of mode was significant $[F(5,120)=$ 7.947, $p=.000, \eta_{\mathrm{p}}^{2}=.249$ ] (for Mode $_{C}, M=73.1 \%$, $S D=16.6$; for Mode $_{1}, M=57.9 \%, S D=10.6$; for Mode $_{3}, M=63.1 \%, S D=13.3$; for Mode $_{4}, M=59.0 \%$, $S D=10.7$; for Mode $_{6+7}, M=59.8 \%, S D=12.5$; for Mode $\left._{8-20}, M=61.3 \%, S D=13.2\right)$. The pairwise comparisons showed that anticipation in the control condition $\left(\right.$ Mode $\left._{C}\right)$ differed from all other conditions (Mode 1, Mode $_{3}$, Mode $_{4}$, Mode $\left._{6+7}, M_{\text {ode }}{ }_{8-20}\right)$, which did not differ significantly from each other. The anticipation accuracy in each condition (i.e., Mode ${ }_{C}$, Mode $_{1}$, Mode $_{3}$, Mode $_{4}$, Mode $_{6+7}$, Mode $_{8-20}$ ) was above chance (at $\alpha=.05$ ).

The observation that a combination of higher modes (i.e., Modes 6 and 7 and 8-20) allowed for response accuracy above chance came as a surprise. Therefore, we further analyzed the eigenvectors obtained in Experiment 1 in terms of shot direction and depth. We performed ANOVAs with shot direction (2) and shot distance (2) as within-participants factors for Modes 6 to 10 for each marker location (18) and direction (3) (see also Experiment 1). Although the number of significant observations vis-à-vis shot direction decreased with increasing mode, this effect did not disappear.

In sum, the results of Experiments $3 \mathrm{~A}$ and $3 \mathrm{~B}$ indicated that all combinations of modes tested allowed for anticipation above chance. However, anticipation accuracy did not significantly deteriorate relative to the control condition, except for the linear addition of Modes 1 and $3\left(\right.$ Mode $\left._{1+3}\right)$ and Modes 2 and 3 (Mode $2+3)$.

\section{Discussion}

The aim of the present experiment was to identify the mode(s) facilitating anticipation. In both parts (A and B), we found an unexpected significant effect of player. Recall that the simulations were based on the mean eigenvectors and projections found in Experiment 1, and that means and standard deviations from two players were averaged across trials for shot direction and distance. The only differences between the simulations corresponding to these players were in terms of the scaling of the dynamics (i.e., the $T S_{S D}$ ). This scaling may have affected the extent to which observers were able to pick up and use the dynamic information contained in the displays. This find would be consistent with that of Pollick, Fidopiastis, and Braden (2001), who reported that the categorization of tennis stroke styles can be improved when the strokes are spatially exaggerated (at least for some styles).

Unexpectedly, we found no significant effect of skill. However, one must remember that in the present experiment, in which shot direction differences were eliminated by including modes, the amount of shot-direction-specific variance relative to the entire amount of variance was lower than in Experiment 2, in which we displayed modes with shot-direction differences present. This effect may have diminished skill differences and contributed to the lack of a significant skill effect. Regardless, we found that no single dynamic structure contained sufficient information to allow for anticipation that was not different from the control condition, but that the combination of Modes 1 and $3\left(\right.$ Mode $\left._{1+3}\right)$ and Modes 2 and $3\left(\right.$ Mode $\left._{2+3}\right)$ did. The information content of the combination of Modes 1 and $2\left(\right.$ Mode $\left._{1+2}\right)$, however, appeared to be insufficient to facilitate effective anticipation. These results suggest that the informational value of the dynamics is not simply a matter of the amount of variance accounted for by the dynamics. The linear addition of directional information contained in Modes 1 and 3, as well as in Modes 2 and 3, structures the high-dimensional motion pattern and thereby renders it sufficiently informational to allow for accurate anticipation.

The response accuracy was significantly above chance in each condition. Although we had anticipated this result for the first three modes, we were somewhat surprised to find it for the higher modes. The additional analysis of the eigenvectors, however, indicated that differences in shot direction were present at least up to the 10th mode. Notwithstanding the small amount of variance covered by the higher modes, shot-specific differences in these modes can be picked up and used, at least when only these differences are present. It appears that, even though at the cost of accuracy, the visual system is sensitive and flexible in picking up the information contained in those modes when constrained in a specific manner. Indications of such perceptual sensitivity and flexibility have been hinted at in previous research. For instance, whereas evidence suggests that in three-ball cascade juggling, the zenith of the ball trajectories is the most informative part of the trajectory for sustaining juggling, other portions of the trajectories are sufficiently informative to enable effective performance (Huys \& Beek, 2002; Huys et al., 2004; van Santvoord \& Beek, 1994). Also, people are able to detect small differences in trajectory forms of oscillators, including asymmetry and symmetrical peakening or flattening - changes that may be indicative of biological movement (Muchisky \& Bingham, 2002). In sum, although each tested mode or combination of modes allowed for anticipation above chance, only the linear combination of directional information in Modes 1 and 3 and in Modes 2 and 3 allowed for statistically undisturbed anticipation. 

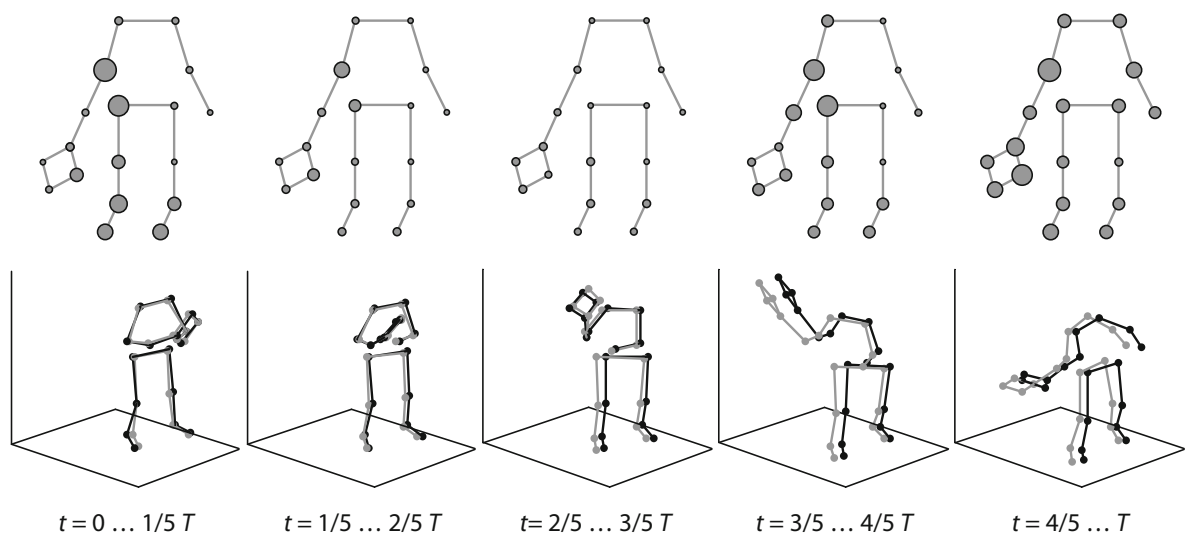

Figure 8. The stick figures in the upper row represent the root-mean square (RMS) difference between the time evolutions of the inside-out and cross-court shots, as a function of marker location and normalized time (see text). The marker size corresponds to the RMS. Each panel represents the RMS in a time window of one fifth of the entire shot duration $(T)$; time increases from the left to right panel. The stick figures in the lower row represent "snapshots" of the inside-out shot (black) and cross-court shot (gray) at $10 \%, 30 \%, 50 \%, 70 \%$, and $90 \%$ of the normalized time (i.e., in the middle of the corresponding time windows) (see text).

In Experiment 1, we showed that a few modes accounted for most of the variance underlying tennis shot executions, and that the variance, its variability across shots, and the shot-direction-specific dynamic differences were distributed heterogeneously across the body and racket in a mode-specific manner. Experiments 2 and 3 suggested that the information underpinning anticipation of tennis shot direction resides in the first three modes. That is, we can localize and quantify the perceptually relevant dynamic differences between both shot directions throughout their execution. To that aim, we constructed an inside-out and cross-court shot based on the first three principal modes by computing the product of the time evolutions and the eigenvectors of Modes 1 to 3; that is, $\mathbf{q}(t)=\xi_{k}(t) \cdot v_{i}^{k}$, for $k=1$ to 3 , and $i=1 \ldots 54$ (see also Equation 1). For each marker (18), we calculated the root-mean square (RMS) difference between the inside-out and cross-court shot as a function of time according to the equation at the bottom of the page (the subscripts IO and XC indicate the insideout and cross-court shots, respectively) and determined its mean in five consecutive time windows, each with a duration of $20 \%$ of the (normalized) shot (see Figure 8, upper panel). We find it important to point out that the RMS represents a difference score in terms of the structure of the trajectories, not in terms of "real-world" coordinates. To further visualize the results (see Figure 8, lower panel), we computed the corresponding "real-world" coordinates [by multiplying each marker's time series $\mathbf{q}_{i}(t)$ by its corresponding standard deviation and adding its mean; see also above] and plotted "snapshots" of the inside-out and cross-court shot at the middle of each time window. Two observations are apparent from Figure 8 (upper panel). First, at the beginning of the shots, differences in the time evolutions prevail in the right lower body and the right hip and elbow. Decreasing in the middle part of shot execution, the dynamic shot differences start to increase at the right side of the body and then spread across the entire body toward ball contact (at $t=T$ ), although a slight right-side dominance remains. Second, regardless of the heterogeneous distribution in space and time, differences in shot direction are, to some degree, present across the entire body and racket throughout the entire shot. These results are roughly in line with those of previous research involving the recording of eye movement data; expert observers typically adopt a proximal-to-distal visual scanning path (i.e., from the hips and shoulders to the arm and racket, respectively). However, it appears that only a limited selection of areas in which shot-direction differences are present are gazed at, if we accept the assumption that optical information pickup can be equated with gaze direction (Williams et al., 1999).

\section{GENERAL DISCUSSION}

In the present article, we have examined the dynamic structure of different tennis shots, as well as any shotspecific differences. We found that tennis shots could be effectively approximated by a few dynamic structures. Following this preliminary analysis, we examined whether the information contained in these structures could be picked up and used to anticipate shot direction by skilled and less skilled tennis players in two experiments. We discovered that a low-dimensional (3-D) representation of the shots allowed for undisturbed anticipation, and that the information underlying anticipation performance is not contained in a single dynamic structure, but in a linear combination of these three structures. In the following, we discuss the theoretical implications of these results for

$$
\operatorname{RMS}(t)=\sqrt{\left[x_{\mathrm{IO}}(t)-x_{\mathrm{XC}}(t)\right]^{2}+\left[y_{\mathrm{IO}}(t)-y_{\mathrm{XC}}(t)\right]^{2}+\left[z_{\mathrm{IO}}(t)-z_{\mathrm{XC}}(t)\right]^{2}}
$$


understanding the control of complex motor skills, the perception of biological motion, and anticipation skill. We interpret the findings from the perspective of coordination dynamics, which we therefore briefly address first.

\section{Macroscopic Structures and Information}

Coordination dynamics has its conceptual and methodological roots in Haken's synergetics $(1977,1996)$, which is, broadly speaking, concerned with qualitative changes in complex open systems (i.e., with the spontaneous formation and, hence, characterization of spatial, temporal, and functional patterns around phase transitions). Complex open systems that are far from thermal equilibrium may organize themselves by forming coherent, ordered spatial and temporal patterns and/or structures due to the weakly nonlinear interactions among their numerous modes. Although the occurrence of such patterns reflects the emergence of covariation among modes at the microscopic level, the resulting macroscopic patterns may be described by a small number of so-called macroscopic structures or order parameters. In other words, a highdimensional system may be summarized by a few order parameters that capture the system's state, implying a huge reduction of information.

In the study of coordinated human movement, it is well established that the relative phase between two oscillating limbs represents, or has the characteristics of, an order parameter (cf. Haken, Kelso, \& Bunz, 1985; Kelso, 1981, 1984, 1995). Less well established, but most relevant for our present purposes, is the conjecture that the visual recognition of dynamic patterns may proceed through the extraction of macroscopic motion structures such as the modes $\mathbf{v}^{k}$ distilled by the Karhunen-Loève expansion, precisely because those modes represent the low-dimensional information contained in dynamic patterns (Haken, 1996, 2000, 2004). In Haken's own words, "The modes ... contain all the information needed for the reconstruction of the pattern. The coefficients with the largest $\lambda_{j}$ contain most of the information about the pattern vector q. ... All the discriminatory information must be carried by the coefficients $\xi_{j} \ldots$.." (2000, p. 158). In the present context, the coefficients $\xi_{j}$ are dynamic - that is, time-varying, $\xi_{j}=\xi_{k}(t)$ (see also Haken, 2000; Haken, Kelso, Fuchs, \& Pandya, 1990). Although principal components or modes cannot and should not be equated with order parameters - since the latter are intrinsically linked to qualitative changes in pattern formation (i.e., to phase transitions) - PCA and related techniques provide a convenient tool for identifying and testing the presence of order parameters around phase transitions because, in principle, they allow one to capture the information reduction that is inherent to order parameters.

\section{The Control of Tennis Shots}

A recurrent theme in motor control research is Bernshteǐn's (1967) so-called degrees-of-freedom problem (see also Turvey, 1990), the problem of how the numerous elements that are involved in the execution of an action are controlled. A solution to this problem has been sought in terms of synergies, or coordinative structures, which, from the perspective of coordination dynamics, are viewed as coherent macroscopic spatiotemporal patterns generated under nonequilibrium constraints in open systems (Kugler, Kelso, \& Turvey, 1980). In the present study, we found that three structures accounted for almost $90 \%$ of the variance, implying that the dynamic structure underlying passing shots is low dimensional. However, there were no indications that different shot types (in terms of distance and direction) were executed in a qualitatively different manner: No single orthogonal (i.e., independent) mode was uniquely associated with a specific shot type.

The variability of the eigenvector coefficients was distributed unevenly across different body areas and trials, suggesting that certain muscular-skeletal linkages are controlled to a greater extent, or at least more consistently so, than others. In addition, the average covariance between the projections of the first three modes of a specific shot condition was close to 1 (see Experiment 1), which hints at a large degree of similarity in the control structure underlying tennis-shot execution across trials within participants. It appears that the synergies or coordinative structures governing tennis strokes are low-dimensional temporal organizations with a high degree of similarity in their global structure, even though the degree to which their constituent modes are controlled across performances is distributed heterogeneously.

\section{Dynamic Structures Underlying Anticipation}

In Experiments 2 and 3, we examined the extent to which observers were able to pick up and use the information contained in the modes identified in Experiment 1 . We found that several dynamic structures were informative. Participants were able to pick up and use lowdimensional structures for anticipation, but the presentation of specific, linear combinations of structures alone allowed for accurate performance. The high accuracy scores in Experiment 2-when we presented three, four, or five modes, but not fewer-indicated that dynamic information was sufficient for attaining high performance levels in the anticipation of shot direction. In Experiment 3, we found that the response accuracy that was based on linear addition of Modes 1 and $3\left(\operatorname{Mode}_{1+3}\right)$, as well as of Modes 2 and 3 (Mode $2+3$ ) - but not of Modes 1 and 2 $\left(M_{o d e}{ }_{1+2}\right)$ - did not differ significantly from the control condition $\left(\right.$ Mode $\left._{C}\right)$. In addition, we found that shot differences in the higher modes allowed for anticipation above chance, but only when the information in the most important modes was eliminated (compare Experiments 2 and 3). In combination, these results indicated that although the representations of tennis shots needed to account for a large portion of the variance (roughly $90 \%$ ) in order to allow for accurate anticipation, both the amount of variance accounted for and the structure of those representations, as determined by the specific combination of orthogonal modes, mattered.

Having identified the dynamic structures underlying the anticipation of shot direction, what can we say about the corresponding information? Focusing on Modes 1 to 3 , we found corresponding variance across the entire 
body, little to moderate trial-to-trial variability (relative to that in Modes 4 and higher), and consistent shot-direction differences at all locations, even though the distribution of these modes was heterogeneous (see Figures 4, 5, and 6, respectively). These observations are reflected in the RMS shot differences that correspond with the linear addition of the first three modes (see Figure 8). Associated with each mode is a specifically structured variance (see Figure 3 ) that is distributed unevenly across the various body areas and racket (represented by the eigenvector coefficients). We find it important to point out that in each context-specific action, the structured variance and the eigenvector coefficients' distribution are two sides of the same coin; they constrain each other, so that neither can be altered without affecting the other.

The perceptual importance of the variance structure has been shown empirically by Troje (2002; see also Westhoff \& Troje, 2007) and indirectly by Johansson (1973). Troje used PCA in his study of gait-mediated gender recognition. He showed that the third mode, which oscillated at twice the frequency of the first two modes, was more important for gait-mediated gender recognition than the second mode was, even though the latter was associated with greater variance. In a similar vein, Johansson (1973) found that subtracting or adding a common motion mode did not affect the observers' recognition of walking. In our view, this was the case because the structures carrying the information for walking were structurally unaltered by these manipulations. In the present case, the fact that all contributing body and racket areas share the three perceptually relevant time evolutions to a varying degree indicates that shot-directionspecific invariance resides in the spatiotemporal relations that are defined by the linear addition of the first three modes across the corresponding areas (see Figure 8, upper panel). Dynamic differences are distributed unevenly in space and time, and their omnipresence suggests that attempts to strictly localize the information underlying shot direction anticipation are inherently arbitrary. However, this does not necessarily imply that observers pick up and use the information from all possible locations.

Overall, the present results, like those of Troje (2002; Westhoff \& Troje, 2007), support the premise that the macroscopic structures that summarize a system's state are informational. A few structures captured most of the tennis-shot execution's variance. It appeared that the shotdirection-specific differences inherent in the linear combination of the first three structures carried the information needed to allow for undisturbed shot-direction anticipation. Following Haken (2000), we conjecture that these macroscopic coordinative structures are readily observable from the kinematics and constitute informational quantities precisely because they capture the system's state. That is, pattern recognition is based on the variable(s) that (phenomenologically) capture(s) coordination patterns.

\section{Perceptual Expertise in Anticipation}

To date, most researchers have relied on spatial-temporal occlusion methods and eye movement recordings to uncover the mechanisms mediating skill differences in this type of task (Abernethy \& Russell, 1987; Ward et al., 2002; Wil- liams et al., 2002). The findings from this body of work suggest that expertise reveals itself in the ability to pick up and use information that is generated earlier in the unfolding action (typically generated at body areas proximal to the end effector), as well as in an increased ability to pick up and make full use of the available information. These results have often been (re)phrased in terms of experts' being able to pick up subtle cues (Shim et al., 2005; Ward et al., 2002). The present results extend and refine some previous suggestions, but they contradict others. Our work strongly suggests that subtle cues may readily be substituted with lowdimensional dynamic information. What distinguished the skilled from less skilled players in Experiment 2 was not the ability to pick up the available information, but rather to pick up the relevant low-dimensional (i.e., invariant) information. In fact, when available, the surplus (i.e., high-dimensional) information present in the optical array was not used in an additive fashion (see also, Bruno \& Cutting, 1988). The present statistical results do not allow any firm conclusions, but they do suggest that additional (high-dimensional) information tended to lead the less skilled players astray. By hypothesis, the less skilled players may not (yet) have acquired or refined the ability to pick up the invariance in the complex high-dimensional motion patterns that underwrites perceptual expertise in anticipation.

In the above regard, it has been suggested in the area of motor control that learning involves a reduction in the dimensionality of the dynamic structure underlying the execution of actions (Haken, 1996; Huys et al., 2004; Mitra, Amazeen, \& Turvey, 1998). It should be emphasized, however, that the implicit assumption that a reduced dimensionality necessarily implies reduced control is open to criticism (Longstaff \& Heath, 2003; Newell \& Vaillancourt, 2001). The present results suggest, or at least are compatible with the understanding, that becoming skilled at perceiving biological motion patterns may involve a reduction of information of the kind suggested in synergetics. In fact, previous research on tennis-shot anticipation suggests that experts visually scan a significantly larger part of the opponent's unfolding action, referred to as a holistic visual search approach, than do nonexperts (Ward et al., 2002; Williams et al., 2002). Since shot-direction-specific invariance is distributed across the entire body, it may be that the more holistic search serves to pick up the invariance to a fuller extent than search methods used by nonexperts do. In sum, the present results suggest that learning to anticipate and, maybe in a broader sense, to perceive biological motion involves distinguishing the low-dimensional dynamics (i.e., invariance) in high-dimensional displays.

\section{Concluding Remarks}

In the present article, we have shown that the execution of tennis shots is based on a low-dimensional dynamic structure, and that these dynamics inform players about shot direction. Coordination dynamics constitutes an appealing theoretical framework for accounting for this finding because it views and treats pattern production and pattern recognition as two sides of the same coin. Theoretical interpretations aside, the present results readily raise a variety of research questions of both basic and 
applied significance that could be fruitfully addressed within the present methodological framework. For instance, could perceptual learning be facilitated through the use of low-dimensional displays, or perhaps through the exaggeration of the dynamics, thereby caricaturing the actions to be learned? Also, the dynamic structure is defined over whole-body movements, which suggests (but does not dictate) that observers use "globally" rather than "locally" defined dynamics. The present methodology allows for a thorough investigation of the issue of local versus global perception in anticipation (cf. Haken, 2004; Watanabe \& Kikuchi, 2006). Finally, in the present study, we used PCA to decompose the movement data into a smaller set, implicitly assuming a correspondence with the perceptual system's functioning at some level. Although this approach worked well, there is no a priori reason to assume that pattern recognition should be organized linearly, according to maximization of variance along orthogonal modes; a variety of other, linear as well as nonlinear, reduction methods may obtain a better correspondence. This example only highlights the fact that much work remains to be done, using the theoretical and methodological framework adopted in this article to unravel how individuals anticipate and perceive biological motion.

\section{AUTHOR NOTE}

Correspondence concerning this article should be addressed to R. Huys, UMR 6233 Institut des Sciences du Mouvement "Etienne-Jules Marey," Université de la Méditerranée, Faculté des Sciences du Sport, CP 910, 163 Av. de Luminy F-13288 Marseille CEDEX 09, France (e-mail: raoul .huys@univmed.fr).

\section{REFERENCES}

Abernethy, B. (1990). Anticipation in squash: Differences in advance cue utilization between expert and novice players. Journal of Sports Sciences, 8, 17-34.

Abernethy, B., Gill, D. P., Parks, S. L., \& Packer, S. T. (2001) Expertise and the perception of kinematic and situational probability information. Perception, 30, 233-252.

Abernethy, B., \& Russell, D. G. (1987). The relationship between expertise and visual search strategy in a racquet sport. Human Movement Science, 6, 283-319.

BERNSHTEǏN, N. A. (1967). The co-ordination and regulation of movements. Oxford: Pergamon.

Bruno, N., \& Cutting, J. E. (1988). Minimodularity and the perception of layout. Journal of Experimental Psychology: General, 117, $161-170$.

Daffertshofer, A., Lamoth, C. J. C., Meijer, O. G., \& Beek, P. J. (2004). PCA in studying coordination and variability: A tutorial. Clinical Biomechanics, 19, 415-428.

Dittrich, W. H., Troscianko, T., Lea, S. E. G., \& Morgan, D. (1996) Perception of emotion from dynamic point-light displays represented in dance. Perception, 25, 727-738.

Haken, H. (1977). Synergetics: An introduction. Nonequilibrium phase transitions and self-organization in physics, chemistry, and biology. Berlin: Springer.

Haken, H. (1996). Principles of brain functioning: A synergetic approach to brain activity, behavior, and cognition. Berlin: Springer.

HAKEN, H. (2000). Information and self-organization: A macroscopic approach to complex systems. Berlin: Springer.

HaKen, H. (2004). Synergetic computers and cognition: A top-down approach to neural nets. Berlin: Springer.

HaKen, H., Kelso, J. A. S., \& Bunz, H. (1985). A theoretical model of phase transitions in human hand movements. Biological Cybernetics, 51, 347-356.
Haken, H., Kelso, J. A. S., Fuchs, A., \& Pandya, A. S. (1990). Dynamic pattern recognition of coordinated biological motion. Neural Networks, 3, 395-401.

HuYs, R., \& BEEK, P. J. (2002). The coupling between point-of-gaze and ball movements in three-ball cascade juggling: The effects of expertise, pattern and tempo. Journal of Sports Sciences, 20, 171-186.

Huys, R., Daffertshofer, A., \& Beek, P. J. (2004). Multiple time scales and subsystem embedding in the learning of juggling. Human Movement Science, 23, 315-336.

Johansson, G. (1973). Visual perception of biological motion and a model for its analysis. Perception \& Psychophysics, 14, 201-211.

Johansson, G. (1976). Spatio-temporal differentiation and integration in visual motion perception. Psychological Research, 38, 379-393.

KELSO, J. A. S. (1981). On the oscillatory basis of movement. Bulletin of the Psychonomic Society, 18, 63.

Kelso, J. A. S. (1984). Phase transitions and critical behavior in human bimanual coordination. American Journal of Physiology, 246(6, Pt. 2), R1000-R1004.

KeLso, J. A. S. (1995). Dynamic patterns: The self-organization of brain and behavior. Cambridge, MA: MIT Press.

Kugler, P. N., Kelso, J. A. S., \& Turvey, M. T. (1980). On the concept of coordinative structures as dissipative structures: I. Theoretical lines of convergence. In G. E. Stelmach \& J. Requin (Eds.), Tutorials in motor behavior (pp. 3-47). Amsterdam: North-Holland.

Longstaff, M. G., \& Heath, R. A. (2003). The influence of motor system degradation on the control of handwriting movements: A dynamical systems analysis. Human Movement Science, 22, 91-110.

Mitra, S., Amazeen, P. G., \& Turvey, M. T. (1998). Intermediate motor learning as decreasing active (dynamical) degrees of freedom. Human Movement Science, 17, 17-65.

Muchisky, M. M., \& Bingham, G. P. (2002). Trajectory forms as a source of information about events. Perception \& Psychophysics, 64, 15-31.

Newell, K. M., LiU, Y.-T., \& Mayer-Kress, G. (2001). Time scales in motor learning and development. Psychological Review, 108, 57-82.

Newell, K. M., \& Vaillancourt, D. E. (2001). Dimensional change in motor learning. Human Movement Science, 20, 695-715.

Pollick, F. E., Fidopiastis, C., \& Braden, V. (2001). Recognising the style of spatially exaggerated tennis serves. Perception, 30, 323-338.

Post, A. A., Daffertshofer, A., \& Beek, P. J. (2000). Principal components in three-ball cascade juggling. Biological Cybernetics, 82, 143-152.

RunEson, S. (1983). On visual perception of dynamic events (Acta Universitatis Upsaliensis: Studia Psychologica Upsaliensa). Stockholm: Almqvist \& Wiksell. (Original work published 1977)

Runeson, S., \& Frykholm, G. (1981). Visual perception of lifted weight. Journal of Experimental Psychology: Human Perception \& Performance, 7, 733-740.

Runeson, S., \& FryKholm, G. (1983). Kinematic specification of dynamics as an informational basis for person-and-action perception: Expectation, gender recognition, and deceptive intention. Journal of Experimental Psychology: General, 112, 585-615.

Scholz, J. P., \& SchönER, G. (1999). The uncontrolled manifold concept: Identifying control variables for a functional task. Experimental Brain Research, 126, 289-306.

Shim, J., Carlton, L. G., Chow, J. W., \& Chae, W.-S. (2005). The use of anticipatory visual cues by highly skilled tennis players. Journal of Motor Behavior, 37, 164-175.

Smeeton, N. J., Williams, A. M., Hodges, N. J., \& Ward, P. (2005). The relative effectiveness of various instructional approaches in developing anticipation skill. Journal of Experimental Psychology: Applied, 11, 98-110.

Strogatz, S. H. (1994). Nonlinear dynamics and chaos: With applications to physics, biology, chemistry, and engineering. Reading, MA: Addison-Wesley.

Troje, N. F. (2002). Decomposing biological motion: A framework for analysis and synthesis of human gait patterns. Journal of Vision, 2, 371-387.

Turvey, M. T. (1990). Coordination. American Psychologist, 45, 938953.

van Santvoord, A. A. M., \& BeeK, P. J. (1994). Phasing and the pickup of optical information in cascade juggling. Ecological Psychology, 6, 239-263 
Ward, P., Williams, A. M., \& Bennett, S. J. (2002). Visual search and biological motion perception in tennis. Research Quarterly for Exercise \& Sport, 73, 107-112.

WATANABE, O., \& KIKUCHI, M. (2006). Hierarchical integration of individual motions in locally paired-dot stimuli. Vision Research, 46, 82-90.

Westhoff, C., \& Troje, N. F. (2007). Kinematic cues for person identification from biological motion. Perception \& Psychophysics, 69, 241-253.

Williams, A. M., Davids, K., \& Williams, J. G. (1999). Visual perception and action in sport. London: Spon.
Williams, A. M., \& Ericsson, K. A. (2005). Perceptual-cognitive expertise in sport: Some considerations when applying the expert performance approach. Human Movement Science, 24, 283-307.

Williams, A. M., Ward, P., Knowles, J. M., \& Smeeton, N. J. (2002). Anticipation skill in a real-world task: Measurement, training, and transfer in tennis. Journal of Experimental Psychology: Applied, 8, $259-270$.

ZAR, J. H. (1996). Biostatistical analysis (3rd ed.). Upper Saddle River, NJ: Prentice Hall.

\section{APPENDIX}

Table A1

ANOVA Results for Significant Effects of Shot Direction of the Eigenvector Coefficients for Modes 1 to 5

\begin{tabular}{|c|c|c|c|c|c|c|c|c|c|c|c|c|c|c|}
\hline \multicolumn{3}{|c|}{ Mode 1} & \multicolumn{3}{|c|}{ Mode 2} & \multicolumn{3}{|c|}{ Mode 3} & \multicolumn{3}{|c|}{ Mode 4} & \multicolumn{3}{|c|}{ Mode 5} \\
\hline & $F(1,5)$ & $\eta_{\mathrm{p}}^{2}$ & & $F(1,5)$ & $\eta_{\mathrm{p}}^{2}$ & & $F(1,5)$ & $\eta_{\mathrm{p}}^{2}$ & & $F(1,5)$ & $\eta_{\mathrm{p}}^{2}$ & & $F(1,5)$ & $\eta_{\mathrm{p}}^{2}$ \\
\hline$\overline{\mathrm{RA}_{x}^{\mathrm{B}}}$ & $70.754^{* * * *}$ & .934 & $\mathrm{SH}_{x}^{\mathrm{R}}$ & $59.886^{* * *}$ & .923 & $\mathrm{SH}_{x}^{\mathrm{R}}$ & $47.794^{* * *}$ & .905 & $\mathrm{SH}_{x}^{\mathrm{L}}$ & $18.516^{* *}$ & .787 & $\mathrm{RA}_{x}^{\mathrm{T}}$ & $28.137^{* * * *}$ & .849 \\
\hline $\mathrm{EL}_{x}^{\mathrm{R}}$ & $56.073^{* * *}$ & .918 & $\mathrm{HI}_{x}^{\mathrm{R}}$ & $29.082^{* * *}$ & .853 & $\mathrm{RA}_{y}^{\mathrm{L}}$ & $21.557^{* *}$ & .812 & $\mathrm{EL}_{y}^{\mathrm{R}}$ & $17.907^{* *}$ & .782 & $\mathrm{HI}_{y}^{\mathrm{R}}$ & $19.664^{* *}$ & .797 \\
\hline $\mathrm{SH}_{x}^{\mathrm{R}}$ & $32.094^{* * *}$ & .865 & $\mathrm{WR}_{y}^{\mathrm{L}}$ & $13.318^{*}$ & .727 & $\mathrm{RA}_{x}^{\mathrm{T}}$ & $18.969^{* *}$ & .791 & $\mathrm{WR}_{x}^{\mathrm{L}}$ & $15.769^{*}$ & .759 & $\mathrm{RA}_{x}^{\mathrm{R}}$ & $16.116^{*}$ & .763 \\
\hline $\mathrm{RA}_{x}^{\mathrm{L}}$ & $23.104^{* *}$ & .822 & $\mathrm{EL}_{x}^{\mathrm{R}}$ & $12.092^{*}$ & .707 & $\mathrm{RA}_{y}^{\mathrm{B}}$ & $18.823^{* *}$ & .790 & $\mathrm{EL}_{x}^{\mathrm{L}}$ & $7.859^{*}$ & .611 & $\mathrm{RA}_{x}^{\mathrm{L}}$ & $15.187^{*}$ & .752 \\
\hline $\mathrm{RA}_{x}^{\mathrm{R}}$ & $21.814^{* *}$ & .814 & $\mathrm{TO}_{x}^{\mathrm{R}}$ & $11.663^{*}$ & .700 & $\mathrm{WR}_{x}^{\mathrm{R}}$ & $18.541^{* *}$ & .788 & & & & $\mathrm{SH}_{y}^{\mathrm{L}}$ & $14.284^{*}$ & .741 \\
\hline $\mathrm{AN}_{y}^{\mathrm{L}}$ & $20.529^{* *}$ & .804 & $\mathrm{AN}_{x}^{\mathrm{R}}$ & $11.632^{*}$ & .699 & $\mathrm{RA}_{x}^{\mathrm{R}}$ & $13.460^{*}$ & .729 & & & & $\mathrm{HI}_{z}^{\mathrm{R}}$ & $13.921^{*}$ & .736 \\
\hline $\mathrm{TO}_{y}^{\mathrm{L}}$ & $20.365^{* *}$ & .803 & $\mathrm{SH}_{z}^{\mathrm{L}}$ & $9.522^{*}$ & .656 & $\mathrm{RA}_{y}^{\mathrm{R}}$ & $13.166^{*}$ & .725 & & & & $\mathrm{RA}_{x}^{\mathrm{B}}$ & $10.916^{*}$ & .686 \\
\hline $\mathrm{WR}_{x}^{\mathrm{R}}$ & $18.541^{* *}$ & .788 & $\mathrm{HI}_{z}^{\mathrm{L}}$ & $9.367^{*}$ & .652 & $\mathrm{RA}_{y}^{\mathrm{T}}$ & $13.029^{*}$ & .723 & & & & $\mathrm{SH}_{x}^{\mathrm{L}}$ & $9.949^{*}$ & .666 \\
\hline $\mathrm{RA}_{x}^{\mathrm{T}}$ & $11.986^{*}$ & .706 & $\mathrm{HI}_{y}^{\mathrm{R}}$ & $9.367^{*}$ & .652 & $\mathrm{AN}_{x}^{\mathrm{R}}$ & $8.981^{*}$ & .642 & & & & $\mathrm{SH}_{z}^{\mathrm{L}}$ & $8.975^{*}$ & .642 \\
\hline $\mathrm{HI}_{x}^{\mathrm{L}}$ & $10.136^{*}$ & .670 & $\mathrm{KN}_{x}^{\mathrm{R}}$ & $9.214^{*}$ & .648 & $\mathrm{RA}_{x}^{\mathrm{L}}$ & $7.000^{*}$ & .583 & & & & $\mathrm{AN}_{z}^{\mathrm{R}}$ & $8.714^{*}$ & .635 \\
\hline $\mathrm{HI}_{x}^{\mathrm{R}}$ & $9.742^{*}$ & .661 & $\mathrm{EL}_{y}^{\mathrm{L}}$ & $9.067^{*}$ & .645 & & & & & & & $\mathrm{WR}_{x}^{\mathrm{L}}$ & $8.440^{*}$ & .628 \\
\hline $\mathrm{TO}_{y}^{\mathrm{R}}$ & $7.021^{*}$ & .584 & & & & & & & & & & $\mathrm{KN}_{z}^{\mathrm{R}}$ & $7.626^{*}$ & .604 \\
\hline $\mathrm{AN}_{z}^{\mathrm{R}}$ & $6.981^{*}$ & .583 & & & & & & & & & & & & \\
\hline
\end{tabular}

Note- The marker location is denoted by its first two letters (RA, racket; SH, shoulder; EL, elbow; WR, wrist; HI, hip; KN, knee; AN, ankle; $\mathrm{TO}$, toe). The superscript denotes the body side/racket part (L, left; R, right; T, top; B, bottom). The subscript denotes the movement direction $(x, y, z) . \quad{ }^{*} p<.05 .{ }^{* *} p<.01 .{ }^{* * *} p<.005 .{ }^{* * * *} p<.001$.

Table A2

ANOVA Results for Significant Effects of Shot Distance and Shot Direction $\times$ Shot Distance Interactions of the Eigenvector Coefficients for Modes 1 to 5

\begin{tabular}{|c|c|c|c|c|c|c|c|c|c|c|c|c|c|c|c|}
\hline \multirow[b]{2}{*}{ Effect } & \multicolumn{3}{|c|}{ Mode 1} & \multicolumn{3}{|c|}{ Mode 2} & \multicolumn{3}{|c|}{ Mode 3} & \multicolumn{3}{|c|}{ Mode 4} & \multicolumn{3}{|c|}{ Mode 5} \\
\hline & & $F(1,5)$ & $\eta_{\mathrm{p}}^{2}$ & & $F(1,5)$ & $\eta_{\mathrm{p}}^{2}$ & & $F(1,5)$ & $\eta_{\mathrm{p}}^{2}$ & & $F(1,5)$ & $\eta_{\mathrm{p}}^{2}$ & & $F(1,5)$ & $\eta_{\mathrm{p}}^{2}$ \\
\hline \multirow[t]{3}{*}{ Shot distance } & & & & $\mathrm{HI}_{z}^{\mathrm{L}}$ & 11.035 & .688 & \multirow[t]{3}{*}{$\mathrm{TO}_{y}^{\mathrm{R}}$} & \multirow[t]{3}{*}{15.712} & \multirow[t]{3}{*}{.759} & $\mathrm{WR}_{y}^{\mathrm{R}}$ & 7.404 & .597 & \multirow[t]{3}{*}{$\mathrm{EL}_{y}^{\mathrm{L}}$} & \multirow[t]{3}{*}{13.275} & \multirow[t]{3}{*}{.726} \\
\hline & & & & $\mathrm{HI}_{y}^{\mathrm{R}}$ & 11.035 & .688 & & & & $\mathrm{RA}_{x}^{\mathrm{T}}$ & 7.146 & .588 & & & \\
\hline & & & & $\mathrm{AN}_{z}^{\mathrm{L}}$ & 10.215 & .671 & & & & & & & & & \\
\hline Shot direction $\times$ & & & & & & & & & & & & & & & \\
\hline shot distance & $\mathrm{KN}_{y}^{\mathrm{L}}$ & 8.382 & .626 & $\mathrm{RA}_{x}^{\mathrm{B}}$ & 9.921 & .665 & & & & $\mathrm{KN}_{z}^{\mathrm{R}}$ & 10.084 & .669 & $\mathrm{RA}_{x}^{\mathrm{T}}$ & 6.646 & .571 \\
\hline
\end{tabular}

(Manuscript received January 24, 2007;

revision accepted for publication March 24, 2008.) 